\title{
Planar Nonlinear Galloping of Iced Transmission Lines under Forced Self-Excitation Conditions
}

\author{
Xiaohui Liu $(\mathbb{D}),{ }^{1}$ Shuguang Yang $\left(\mathbb{D},{ }^{2}\right.$ Chuan Wu $\mathbb{D}^{3},{ }^{3}$ Ming Zou $\left(\mathbb{D},{ }^{2}\right.$ Guangyun Min $\left(\mathbb{D},{ }^{2}\right.$ \\ Ceshi Sun $\left(\mathbb{i},{ }^{1}\right.$ and Mengqi Cai $\mathbb{B}^{4}{ }^{4}$ \\ ${ }^{1}$ State Key Laboratory of Mountain Bridge and Tunnel Engineering, Chongqing Jiaotong University, Chongqing 400074, China \\ ${ }^{2}$ School of Civil Engineering, Chongqing Jiaotong University, Chongqing 400074, China \\ ${ }^{3}$ State Grid Henan Electric Power Research Institute, Zhengzhou 450052, China \\ ${ }^{4}$ School of Architecture and Civil Engineering, Chengdu University, Chengdu 610106, China
}

Correspondence should be addressed to Shuguang Yang; 622180970178@mails.cqjtu.edu.cn

Received 24 December 2020; Revised 7 March 2021; Accepted 10 March 2021; Published 23 March 2021

Academic Editor: Sundarapandian Vaidyanathan

Copyright ( 2021 Xiaohui Liu et al. This is an open access article distributed under the Creative Commons Attribution License, which permits unrestricted use, distribution, and reproduction in any medium, provided the original work is properly cited.

In order to study the influence of dynamic wind on the nonlinear galloping characteristics of iced transmission lines, an external excitation load is added to the governing equation of iced transmission lines under the condition of stable wind, and a new forced self-excited system has been established. The frequency-amplitude relationship of the forced self-excited system under weak excitation and strong excitation is obtained by using the multiple-scale method. The principal resonances and superharmonic and subharmonic resonances of the forced self-excited system have also been analyzed. The results show that, in the forced self-excited system under strong excitation, when the excitation frequency is close to the integral and fractional times of the natural frequency, it is easier to produce 1/2-order subharmonic resonance, 2-order superharmonic resonance, and 3-order superharmonic resonance. In addition, numerical techniques provide bifurcation diagrams of different control parameters, which are able to highlight the effects of the simultaneous presence of the sources of excitation. When the control parameters (wind velocity, excitation amplitude, tuning parameter, tension, and Young's modulus) change, the response amplitudes of the principal resonance and harmonic resonance will have multivalues, jump phenomenon, and hardening behavior. The control parameters can be used as a reference for engineering design. More importantly, as a combination of the Duffing equation and the Rayleigh equation, the forced self-excited system also has high theoretical research value.

\section{Introduction}

The cross section of the transmission lines would change from circular cross section to crescent or D-shape cross section under complex climatic conditions such as snow rime, sleet, and freezing rain $[1,2]$. Thus, the iced transmission lines are prone to galloping under wind load [2]. Long-time and continuous galloping of transmission lines will lead to fatigue failure of structures and poses a significant threat to the safety and serviceability of these structures $[2,3]$. In this case, short circuit, frequent tripping, broken strand or wire, and other accidents will be likely to often occur in the transmission line structure $[4,5]$. In order to investigate the galloping of iced transmission lines, Den
Hartog [6] proposed the vertical galloping mechanism, indicating that galloping mainly occurred in the vertical direction. The vertical galloping mechanism is based on the linear theory, and in fact, the span length of transmission lines is much larger than the diameter of transmission lines, so the transmission lines actually belong to the cable structure, which would show obvious nonlinear behaviors when they are affected by gravity and external forces [7]. In addition, like the transmission line, the span of the stay cable is much larger than its diameter. Therefore, both transmission lines and cables belong to the cable structure.

In recent years, many scholars have studied the nonlinear dynamic behavior of cables under external excitation. The cables in stayed bridges had one fixed end, and the other 
end was subjected to harmonic vertical excitation. At first, through the method of experiment and finite element, Lee and Perkins [8] found the pure planar response, internally resonant nonplanar response, and quasi-periodic responses. $\mathrm{Hu}$ and Pai [9] used the 3D motion analysis system in this experiment, and this experiment had observed and studied the subharmonic and superharmonic resonances, perioddoubling bifurcations, hardening nonlinearity effect, and complex traveling vibrations of cables. Under in-plane and out-of-plane harmonic loads, Gattulli et al. [10] used analytical and finite element models to study the modal interaction of cables in both planar and spatial responses and validated the simplifying kinematic assumptions introduced in the analytical models. In [8-10], the nonlinear vibration of the cable was studied by the method of experiment and finite element. However, there is no qualitative analysis on the vibration form of the cable. Next, through the method of perturbation and numerical method, Nielsen and Kirkegaard [11] obtained the approximate solutions of the cable subjected to superharmonic excitation by the averaging method. And Nielsen discussed the influences about combined harmonics and superharmonics' effect on vibration characteristics of the cable, but he did not take the influence of curvature and small deformation of the cable structure into consideration. Srinil and Rega [12] compared nonlinear modal interactions in shallow horizontal cables with kinematically noncondensed vs. condensed modeling, under simultaneous primary external and internal resonances. And nonlinear dynamic configurations and tensions were also examined. The inclined cable is subjected to a uniformly distributed harmonic load; Rega and Srinil [13] solved partial differential equations by using multimode discretization and a second-order multiple-scale method and found that the hybrid modal interactions undergo several kinds of bifurcations and nonlinear phenomena along with meaningful transition from periodic to quasi-periodic and chaotic responses. Luongo and Zulli [14] analyzed the effect of wind flow simultaneously with rain of the stay cable and studied the interactions between in-plane and out-of-plane motions through bifurcation diagrams. The bifurcations and chaotic dynamics of parametrically and externally excited suspended cables were also investigated in [15]. In [12-15], some scholars studied the nonlinear dynamic effects of different modes on the cable, the coupling vibration of the multidegree of freedom of the cable, the bifurcation analysis of the cable, and the quasi-periodic and chaotic responses of the cable. However, the dynamic response of cables, under the nonsteady wind conditions, has not been studied. Moreover, some scholars also studied the aeroelastic instability of stay cables. And the rain-wind-induced vibration [16] and the dry galloping [17] of the stay cable fall under the problems of aeroelastic instability. As is known to all, it is believed that this aeroelastic instability problem is due to the formation and oscillation of a water rivulet on the upper cable surface, which changes the cable aerodynamics and absorbs energy into the system. Vo et al. [18] confirmed that deformations of the stay cable have a significant influence on the bending aeroelastic instability of the structure by experiments. Huang et al. [19] solved the governing equations for the structure of the suspended cable-stayed beam by using the normal form and the multiple-scale method and found deformations of the stay cable have a significant influence on the bending aeroelastic instability of the structure. In [16-19], some scholars studied the rain-windinduced vibration, ice galloping, and dry galloping of the stay cable and the influence of the deformations of the stay cable on the aerodynamic stability of the cable. The transmission line is also a cable structure because the transmission line is easy to icing, resulting in irregular crosssection shape. Therefore, iced transmission lines are prone to galloping under the excitation of wind load. However, the nonlinear vibration characteristics of iced transmission lines under the influence of dynamic wind are rarely studied. Additionally, Zulli and Luongo $[20,21]$ considered the dynamics of two towers exposed to turbulent wind flow and linked by a nonlinear viscous device. They stated the steady component of the wind is responsible for self-excitation, while the turbulent part causes both parametric and external excitations in a specific resonance condition.

Based on the analysis of [8-19], few scholars have studied the influence of dynamic wind on transmission lines. And based on the influence of dynamic wind on the dynamic response of the structure in [20,21], this paper systematically studies the influence of dynamic wind on the nonlinear galloping characteristics of iced transmission lines. The transmission line is a flexible and elastic cable structure. And the governing equations of the transmission line contain quadratic and cubic nonlinear terms [22], in which the quadratic term is related with the initial curvature of the conductor and the cubic term is related with the axial stretching of the conductor $[23,24]$. Therefore, the dynamic wind is added to the governing equation with quadratic and cubic nonlinear restoring force terms to form a new forced self-excited system. The results show that the principal resonance under weak excitation and the increase in wind velocity or excitation amplitude will enhance the hardening behavior and nonlinear characteristics of the system and will have the multivalue and jump phenomenon. Moreover, the results turn out that increasing the tension and Young's modulus properly can reduce the resonance peak value of the principal resonance and harmonic resonance. The conclusions obtained by this paper will be helpful for the nonlinear galloping analysis of iced transmission lines and also give some references to practical engineering. This paper is organized as follows: in Section 2, we present a mathematical formulation of the iced transmission lines. In Sections 3 and 4, the principal resonance and the harmonic resonance are, respectively, discussed by using the method of multiple scales. A detailed summary of the results is presented in Section 5.

\section{Dynamic Model of Iced Transmission Lines}

Most of high-voltage transmission lines can be equivalent to equal-height transmission lines in plain areas. Therefore, single-span equal-height transmission lines with two fixed supports are considered in this paper. The mechanical model of single-span iced transmission lines is 
established as shown in Figure 1, and it is considered that the wind is along the $z$-axis direction as shown in Figure 2. The transmission lines are assumed to be homogenous and linear elastic with negligible torsional, bending, and shear stiffness. Considering the transmission lines have a small initial sag-to-span ratio (less than 1:8) and low static strain, the associated static equilibrium configuration of the transmission lines can be described through the parabola $y=4 d\left[s / L-(s / L)^{2}\right]$ ( $s$ is the curvilinear abscissa; $L$ is the span length; and $d$ is the sag) [25]. The initial static equilibrium configuration of the transmission lines under gravity is $\xi_{1}$, and when the static equilibrium configuration of the transmission lines changes under the action of other external loads, the corresponding dynamic equilibrium configuration becomes $\xi_{2}$.

Figure 1 is the model of singe-span equal-height transmission lines, and in Figure $1, u(s, t)$ and $v(s, t)$ are the displacements measured from the dynamic equilibrium configuration in the $x$-axis and $y$-axis directions, respectively, and $p * \cos (\Omega t)$ is the forced excitation load.

In order to facilitate the study of the aerodynamic loads of iced transmission lines, it is assumed that the iced shape of the transmission line is crescent. Figure 2 is the model of the cross section of iced transmission lines, Figure 2(a) is the physical model of the transmission line cross section, and Figure 2(b) is the force analysis model of the transmission line cross section; $O_{1} z_{2}$ is the axis of symmetry of the cross section of iced transmission lines, $O_{1} z_{1}$ is the direction in which the horizontal wind acts on the transmission line during vibration, $O_{1} z$ is the horizontal axis, $\alpha$ is the wind attack angle, $\alpha_{0}$ is the initial wind attack angle, $\alpha_{\mathrm{t}}$ is the relative wind attack angle, $U$ is the horizontal wind velocity, $U_{0}$ is the relative wind velocity, and $\dot{v}$ is the vertical galloping velocity.

From Figure 2, it can be obtained that

$$
\tan (\alpha)=\frac{\dot{v}}{U} \approx \alpha
$$

The relative wind acts on the iced transmission lines, which results in an air drag $\left(F_{D}\right)$ along the relative wind direction and an upward air lift $\left(F_{L}\right)$ perpendicular to the relative wind direction [26]; therefore, it can be obtained that

$$
F_{y}=F_{L} \cos (\alpha)-F_{D} \sin (\alpha) \text {. }
$$

Considering the small deformation, that is, $\sin (\alpha) \approx \alpha$ and $\cos (\alpha) \approx 1$, equation (2) can be simplified as follows:

$$
F_{y}=F_{L}-\alpha F_{D}
$$

According to fluid-induced vibration theory, the expressions of $F_{L}$ and $F_{D}$ can be listed [26]:

$$
\begin{aligned}
& F_{L}=\frac{1}{2} \rho U^{2} D C_{L}, \\
& F_{D}=\frac{1}{2} \rho U^{2} D C_{D},
\end{aligned}
$$

where $C_{L}$ is the aerodynamic lift coefficient, $C_{D}$ is the aerodynamic drag coefficient, $\rho$ is the air density, and $D$ is the diameter of the transmission line.

Substituting equations (4a) and (4b) into (3), based on Taylor' law, the aerodynamic coefficients in the $y$-axis direction can be fitted with a cubic nonlinear curve:

$$
C_{y}=C_{0}+A^{\prime} \alpha+B^{\prime} \alpha^{3}+C^{\prime} \alpha^{2},
$$

where $C_{0}, A^{\prime}, B^{\prime}$, and $C^{\prime}$ are undetermined coefficients related to the aerodynamic loads.

According to equations (2)-(4a) and (4b), it can be obtained that

$$
F_{y}=\frac{1}{2} \rho U^{2} D C_{y}
$$

Under the continuous excitation of the mean wind, when the inertia force generated by the vibration of the transmission line is greater than the viscous force of the system, the energy of the system will continue to increase. When the energy input from the outside is equal to the energy dissipated by the system, the transmission line will have selfexcited vibration with constant amplitude. However, in practice, dynamic wind is unstable. Based on this concept, the mean wind $(U)$ in Figure 2(b) is replaced by dynamic wind $(\widehat{U})$, and then the iced transmission line is excited by dynamic wind. In fact, dynamic wind $(\widehat{U})$ can be expressed as the superposition of mean wind $(U)$ and fluctuating wind $(\widetilde{u}(t))$ :

$$
\widehat{U}=U+\widetilde{u}(t) .
$$

The harmonic superposition method can be used to simulate the fluctuating wind, and the random process of fluctuating wind can be simulated by spectral decomposition. Then, it can be obtained that

$$
\widehat{U}=U+\sum_{i=1}^{\infty} U_{i} \cos \left(\Omega_{i} t+\phi_{i}\right)
$$

where $U_{i}$ is the amplitude, $\Omega_{i}$ is the circular frequency, and $\phi_{i}$ is the phase difference.

Substituting equation (8) into equation (6), the vertical aerodynamic lift of dynamic wind can be obtained:

$$
F_{y 1}=\frac{1}{2} \rho D \widehat{U}^{2} C_{y}=\frac{1}{2} \rho D\left\{U^{2}+2 U \sum_{i=1}^{\infty} U_{i} \cos \left(\Omega_{i} t+\phi_{i}\right)+\left[\sum_{i=1}^{\infty} U_{i} \cos \left(\Omega_{i} t+\phi_{i}\right)\right]^{2}\right\} C_{y} .
$$




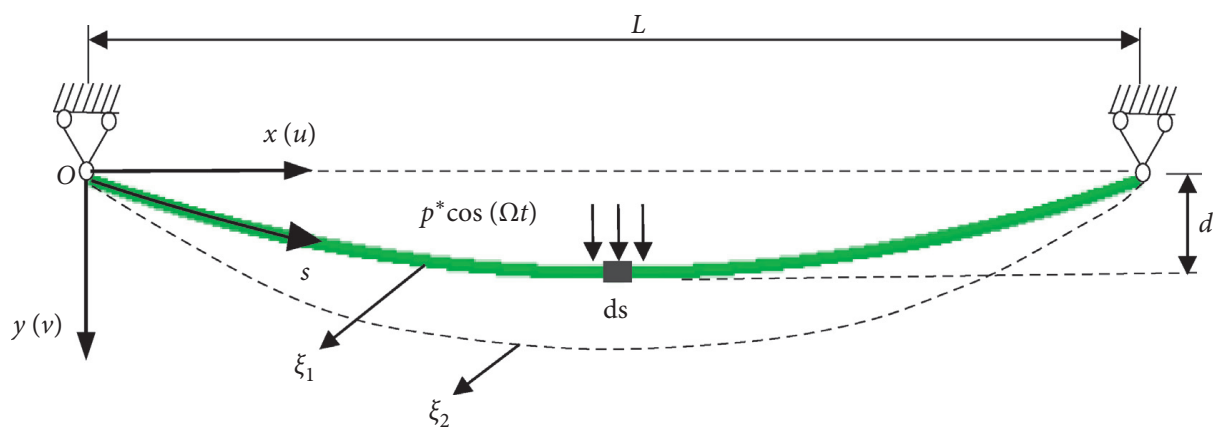

Figure 1: The model of singe-span equal-height transmission lines.

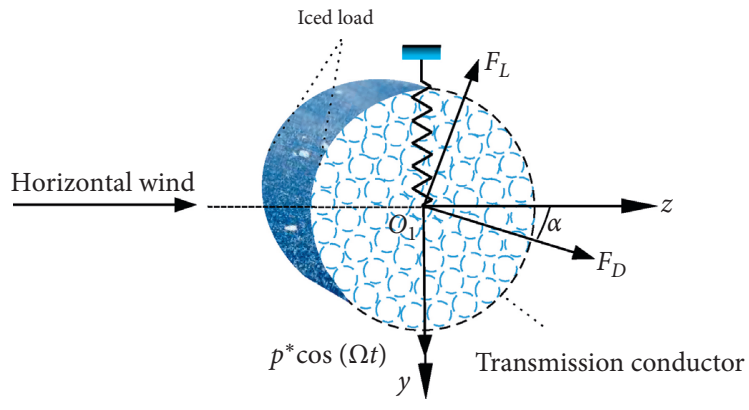

(a)

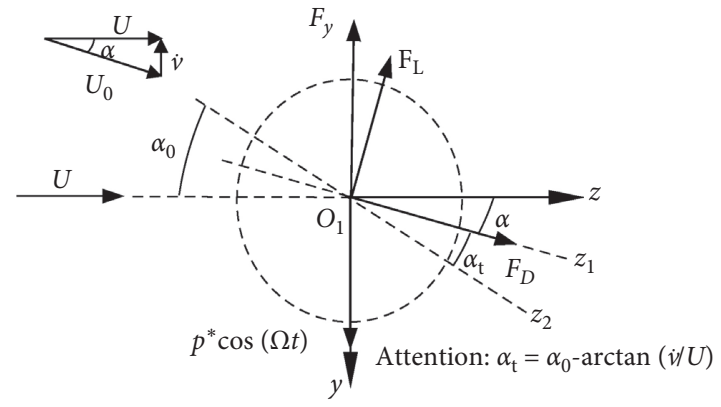

(b)

Figure 2: The model of the cross section of iced transmission lines. (a) Physical model. (b) Force analysis model.

After neglecting the high-order terms of equation (9), it can be obtained that

$$
F_{y 1}=\left[\frac{1}{2} \rho D U^{2}+\rho D U \sum_{i=1}^{\infty} U_{i} \cos \left(\Omega_{i} t+\phi_{i}\right)\right] C_{y} .
$$
that

Substituting equation (5) into (10), it can be obtained

$$
F_{y 1}=\tilde{a} \dot{v}+\widetilde{b} \dot{v}^{3}+\widetilde{c} \dot{v}^{2}+\widetilde{d}+\rho D U \sum_{i=1}^{\infty} U_{i} \cos \left(\Omega_{i} t+\phi_{i}\right) \cdot\left(C_{0}+A^{\prime} \alpha+B^{\prime} \alpha^{3}+C^{\prime} \alpha^{2}\right)
$$

where

$$
\begin{aligned}
& \widetilde{a}=\frac{\rho U D A^{\prime}}{2}, \\
& \widetilde{b}=\frac{\rho D B^{\prime}}{2 U}, \\
& \widetilde{c}=\frac{\rho D C^{\prime}}{2}, \\
& \widetilde{d}=\frac{1}{2} \rho U^{2} D C_{0} .
\end{aligned}
$$

In equation (11), $\rho$ and $D$ denote the air density and the diameter of the transmission line, respectively; $U$ denotes the wind velocity; and $C_{0}, A^{\prime}, B^{\prime}$, and $C^{\prime}$ are related to the aerodynamic force of the iced transmission line, which can be determined by the experiment. The initial wind attack angle and icing shape of the transmission line remain unchanged, and the quasi-static assumption is adopted [27]; then, $C_{0}, A^{\prime}, B^{\prime}$, and $C^{\prime}$ are constant. Since $\rho$ and $D$ are also constants, the coefficient $(\widetilde{c})$ is a constant. The fitting coefficient $(\widetilde{c})$ does not change with the wind velocity $(U)$, so coefficient $(\widetilde{c})$ is also a constant term. And the coefficient $(\widetilde{d})$ does not change with the vertical galloping velocity $(\dot{v})$, so the coefficient $(\widetilde{d})$ is equivalent to adding extra weight on the iced transmission line. And the coefficient $(\widetilde{d})$ has nothing to do with the generation of transmission line galloping, so we can ignore the coefficient $(\widetilde{d})$. Therefore, coefficients $(\widetilde{c})$ and $(\widetilde{d})$ of aerodynamic load are not considered in this paper.

In equation (11), the expression $\left(\rho D U \sum_{i=1}^{\infty} U_{i} \cos \left(\Omega_{i} t+\phi_{i}\right) \cdot\left(A^{\prime} \alpha+B^{\prime} \alpha^{3}+C^{\prime} \alpha^{2}\right)\right)$ is a parametric excitation term in the system, and the expression $\left(\rho D U \sum_{i=1}^{\infty} U_{i} \cos \left(\Omega_{i} t+\phi_{i}\right) \cdot\left(C_{0}\right)\right)$ is an external excitation term in the system. As the coefficient $\left(C_{0}\right)$ is larger than the coefficients $A^{\prime}, B^{\prime}$, and $C^{\prime}$, only the external excitation 
$\left(\rho D U \sum_{i=1}^{\infty} U_{i} \cos \left(\Omega_{i} t+\phi_{i}\right) \cdot\left(C_{0}\right)\right)$ is considered in the following paper. The stable part of dynamic wind is injected into the transmission line to generate self-excited vibration, and the unstable part will cause external excitation [20,21]. Based on this concept, the self-excitation term in equation (11) can be simplified into $F_{y}=\widetilde{a} \dot{v}+\widetilde{b} \dot{v}^{3}$ because it does not consider coefficients $(\widetilde{c})$ and $(\widetilde{d})$ of aerodynamic load; additionally, the external excitation term in equation (11) can be simplified into $F_{y u}=\rho D U \sum_{i=1}^{\infty} U_{i} \cos \left(\Omega_{i} t+\phi_{i}\right) \cdot\left(C_{0}\right)=-p^{*} \cdot \cos (\Omega t)$ without considering the parametric excitation term; then, the vertical aerodynamic force of dynamic wind is

$$
F_{y 1}=F_{y}+F_{y u}=\tilde{a} \dot{v}+\widetilde{b} \dot{v}^{3}-p^{*} \cdot \cos (\Omega t) .
$$

According to the nonlinear vibration theory, when the response contains subharmonic and superharmonic components, the relationship between the restoring force term and the displacement of the system is a nonlinear closed curve, which has obvious nonlinear hysteresis characteristics. The simplified vertical aerodynamic force $F_{y 1}=F_{y}+$ $F_{y u}=\tilde{a} \dot{v}+\widetilde{b} \dot{v}^{3}-p^{*} \cdot \cos (\Omega t)$ of dynamic wind includes the external excitation term $F_{y u}=-p^{*} \cdot \cos (\Omega t)$ and the Rayleigh damping term $F_{y}=\tilde{a} \dot{v}+\tilde{b} \dot{v}^{3}$. The Rayleigh damping, quadratic, and cubic nonlinear restoring force terms constitute the nonlinear hysteresis force of the system.

Den Hartog found that the horizontal amplitude of iced transmission lines was much smaller than the vertical amplitude, indicating that galloping mainly occurred in the vertical direction. Based on this concept, only the galloping in the vertical direction for iced transmission lines is considered in this paper. According to Benedettini and Rega [23], it can be seen that, under the action of dynamic wind, the governing equation of the vertical movement of the transmission line is

$$
\left\{H v^{\prime}+E S\left(y^{\prime}+v^{\prime}\right) \int_{0}^{l}\left[\frac{y^{\prime} v^{\prime}+v^{\prime 2}}{2}\right] \mathrm{d} x\right\}-F_{y}+p^{*} \cdot \cos (\Omega t)-\mu \dot{v}=m \ddot{v},
$$

where $H$ is the tension of the transmission lines, $E$ is Young's modulus of the transmission lines, and $S$ is the crosssectional area of the transmission line. $v^{\prime}$ is the first derivative of the vertical motion function with respect to $x, y^{\prime}$ is the first derivative of the parabolic equation with respect to $x, \dot{v}$ and $\ddot{v}$ are the first derivative and second derivative of the vertical motion function with respect to time $t$, respectively, $\mu$ is the structural damping, and $m$ is the self-weight per unit unstretched length.

The displacement $v(x, t)$ in equation (14) can be written as

$$
\begin{aligned}
v(x, t) & =f(x) q(t), \\
p^{*} & =f_{1}(x) p,
\end{aligned}
$$

where $f(x)=\sin (n \pi x / L)$ is the modal function of iced transmission lines. Let $n=1$, the single-mode discretization is adopted [28], and the research is mainly focused on the first-order mode. In addition, uniform distributed load $\left(p^{*}\right)$ in the vertical direction is considered, $f_{1}(x)=1$ is the modal function of external excitation, and $q(t)$ is the time function.

Based on the Galerkin method, equation (15) is substituted into equation (14) to obtain the nonlinear partial differential equation:

$$
\ddot{q}+\omega^{2} q+c_{1} q^{2}+c_{2} q^{3}+\left(\mu^{*}-c_{3}\right) \dot{q}+c_{4} \dot{q}^{3}=p \cos (\Omega t) .
$$

Equation (16) is the nonlinear galloping governing equation, which includes aerodynamic load and external excitation. The parameters in equation (16) are

$$
\begin{aligned}
\omega^{2} & =\frac{1}{m} \frac{I_{0}}{I_{m}}, \\
c_{1} & =12 \frac{d E S}{m l} \frac{I_{1}}{I_{m}}, \\
c_{2} & =\frac{E S}{2 m l} \frac{I_{2}}{I_{m}}, \\
\mu^{*} & =\frac{\mu}{m}, \\
c_{3} & =\frac{\tilde{a}}{m}, \\
c_{4} & =\frac{\tilde{b}}{m} \frac{I_{b}}{I_{m}}, \\
p & =\frac{p^{*}}{m} \frac{I_{p}}{I_{m}},
\end{aligned}
$$

where

$$
\begin{aligned}
& I_{0}=H \int_{0}^{l} f^{\prime} \cdot f^{\prime} \mathrm{d} x+64 \frac{\mathrm{d}^{2} E S}{l^{5}}\left(\int_{0}^{l} f \mathrm{~d} x\right)^{2}, \\
& I_{1}=\int_{0}^{l} f^{\prime 2} \mathrm{~d} x \int_{0}^{l} f \mathrm{~d} x, \\
& I_{2}=\left(\int_{0}^{l} f^{\prime 2} \mathrm{~d} x\right)^{2}, \\
& I_{m}=\int_{0}^{l} f^{2} \mathrm{~d} x, \\
& I_{b}=\int_{0}^{l} f^{4} \mathrm{~d} x, \\
& I_{p}=\int_{0}^{l} f f_{1} \mathrm{~d} x .
\end{aligned}
$$


Equation (16) contains the first-order and third-order nonlinear damping terms and the second-order and the third-order nonlinear restoring force terms of $q$. The aerodynamic loads in the system include the first-order and third-order terms, which are consistent with the damping term of the Rayleigh function (Rayleigh damping). The aerodynamic loads are presented in the form of the Rayleigh damping in the nonlinear galloping equation. Moreover, the nonlinear restoring force term is consistent with the Duffing function (quadratic and cubic terms). Therefore, equation (16) can be regarded as the combined form of the Duffing equation and the Rayleigh equation. Furthermore, there is an external excitation $(p \cos (\Omega t))$ in equation (16), which excites strong resonance for the system of iced transmission lines. As a result of different frequencies of external excitation, the form of resonance can be divided into the principal resonance of weak excitation and the harmonic resonance of strong excitation.

\section{Principal Resonance of the Weak Nonlinear System}

This section discusses the principal resonance of the forced self-excited system, in which the excitation frequency $\Omega$ is equal to the natural frequency $\omega$. The multiple-scale method is used to obtain the approximate expression of the frequency-amplitude response under weak excitation, where due to the inclusion of quadratic and cubic nonlinear terms, the aerodynamic coefficient of the aerodynamic loads in the system also includes the first-order and third-order terms. It is generally considered that the damping and nonlinear terms have little effect on the system. According to $[10,20]$, the solution form of small parameter $(\varepsilon)$ is

$$
\begin{aligned}
q & =\varepsilon \bar{q}, \\
\mu^{*} & =\varepsilon \bar{\mu}, \\
\dot{q} & =\varepsilon \bar{q}, \\
\dot{q}^{3} & =\varepsilon \bar{q}, \\
p & =\varepsilon \bar{p},
\end{aligned}
$$

where $\bar{q}, \bar{\mu}$, and $\overline{\dot{q}}$ are new variables of the system.

In order to make the equation easy to express, $q, \mu$, and $\dot{q}$ are still used to represent $\bar{q}, \bar{\mu}$, and $\dot{\dot{q}}$. Substituting equation (19) into equation (16), it can be obtain that

$$
\ddot{q}+\omega^{2} q+\varepsilon\left[c_{1} q^{2}+c_{2} q^{3}+\left(\mu^{*}+c_{3}\right) \dot{q}+c_{4} \dot{q}^{3}\right]=\varepsilon p \cos (\Omega t) .
$$

The multiple-scale method has been discussed, and $q$ is set as

$$
q=q_{0}+\varepsilon q_{1}
$$

The partial differential operator symbol and the frequency of external excitation are

$$
\begin{aligned}
D T & =D_{0} T_{0}+\varepsilon\left(D_{0} T_{1}+D_{1} T_{0}\right), \\
\Omega t & =\omega T_{0}+\varepsilon \sigma T_{1} .
\end{aligned}
$$

In equation (22), $\sigma$ is the tuning parameter of the excitation frequency, and substituting equations (21) and (22) into equation (20) and equating coefficients of like powers of $\varepsilon^{n}(n=0,1)$ led to the following linear ordinary equations, respectively:

$$
\begin{aligned}
D_{0}^{2} q_{0}+\omega^{2} q_{0}= & 0 \\
D_{0}^{2} q_{1}+\omega^{2} q_{1}= & -\left[D_{0}^{2} q_{1}+2 D_{0} D_{1} q_{0}+c_{1} q_{0}^{2}+c_{2} q_{0}^{3}\right. \\
& \left.+\left(\mu+c_{3}\right) D_{0} q_{0}+c_{4}\left(D_{0} q_{0}\right)^{3}-p \cos (\Omega t)\right] .
\end{aligned}
$$

In equations (23a) and (23b), $D_{\mathrm{k}}$ is the partial derivative of $T_{\mathrm{k}}$, and the solution of equation (23a) is

$$
q_{0}=A\left(T_{1}\right) e^{i \omega T_{0}}+\bar{A}\left(T_{1}\right) e^{-i \omega T_{0}},
$$

where $A\left(T_{1}\right)$ can be defined as

$$
A\left(T_{1}\right)=\frac{1}{2 a\left(T_{1}\right) \exp \left[i \beta\left(T_{1}\right)\right]},
$$

where $a\left(T_{1}\right)$ and $\beta\left(T_{1}\right)$, respectively, represent the amplitude function and phase function of the system. Substituting equations (24) and (25) into equation (23b), it can be obtained that

$$
D_{1} A=\frac{3 i c_{2}}{2 \omega} A^{2} \bar{A}-\frac{3 c_{4}}{2} \omega^{2} A^{2} \bar{A}-\frac{\mu+c_{3}}{2} A-\frac{i p}{4 \omega} e^{i \sigma T_{1}} .
$$

Substituting equation (25) into equation (26), it can be obtained that

$$
\begin{aligned}
& \dot{a}=\frac{-\left(\mu+c_{3}\right)}{2} a-\frac{3}{8} c_{4} \omega^{2} a^{3}+\frac{p}{2 w} \sin \left(\sigma T_{1}-\beta\right), \\
& \dot{a}=\frac{3 c_{2}}{8 \omega} a^{3}-\frac{p}{2 \omega} \cos \left(\sigma T_{1}-\beta\right) .
\end{aligned}
$$

Introduce $\gamma=\sigma T_{1}-\beta$ to acquire the steady-state solution of the amplitude and phase of equations (27a) and (27b), let $D_{1} A=0$, and obtain the frequency-amplitude equation by eliminating $\gamma$ :

$$
\frac{p^{2}}{4 \omega^{2} a^{2}}=\left(\sigma-\frac{3 c_{2} a^{2}}{8 \omega}\right)^{2}+\left(\frac{\mu+c_{3}}{2}+\frac{3 \omega^{2} c_{4} a^{2}}{8}\right)^{2} .
$$

According to on-site observations, the icing of crescent shaped is a common ice type with galloping. In addition, the parameters of iced transmission lines can be obtained by the experiment. In order to facilitate analysis and comparison, as shown in Table 1, the geometrical parameters, material parameters, and related aerodynamic parameters are cited in [29].

It is necessary to verify the correctness of the approximate solution of the frequency-amplitude equation before discussing the principal resonance of the forced self-excited system. As shown in Figure 3, the tuning parameter $\sigma=0$, and the excitation amplitude $(p)$-response amplitude $(a)$ curve of equation (28) is drawn by mathematical software Maple. In this paper, the curve of Figures 4 and 13 is also drawn by Maple. The actual unit of response amplitude is $m$, 
TABLE 1: The physical parameters of the transmission line.

\begin{tabular}{lccc}
\hline Parameter & Symbol & Unit & Value \\
\hline Tension & $H$ & $\mathrm{~N}$ & 30,000 \\
Span & $L$ & $\mathrm{~m}$ & 125.88 \\
Young's modulus & $E$ & $\mathrm{~N} / \mathrm{mm}^{2}$ & 47803.3 \\
Diameter & $D$ & $\mathrm{M}$ & 0.0286 \\
Mass per unit length & $m$ & $\mathrm{Kg} / \mathrm{m}$ & 2.379 \\
Traverse area & $S$ & $\mathrm{~mm}^{2}$ & 423.24 \\
Air mass density & $\rho$ & $\mathrm{Kg} / \mathrm{m}^{3}$ & 1.2929 \\
Wind velocity & $U$ & $\mathrm{~m} / \mathrm{s}$ & 4.0 \\
Sag & $d$ & $\mathrm{~m}$ & 1.5432 \\
Vertical damping & $\mu$ & - & 0.0005 \\
Aerodynamic parameters & $A^{\prime}$ & - & -0.1667 \\
Aerodynamic parameters & $B^{\prime}$ & - & 8.3581 \\
\hline
\end{tabular}

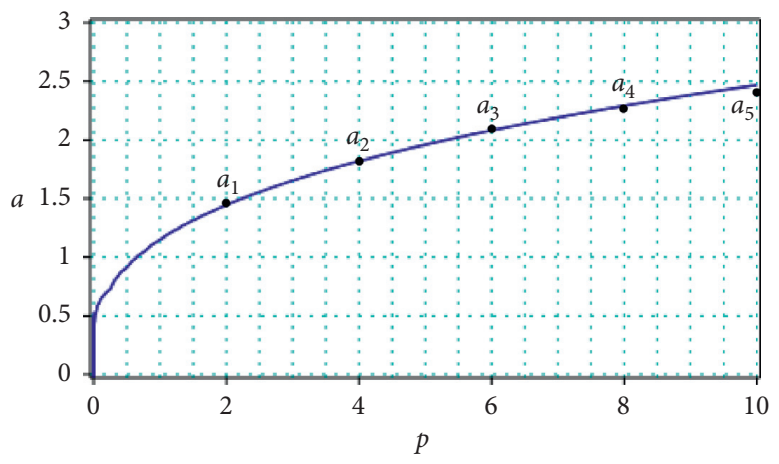

FIGURE 3: The principal resonance curve of excitation amplitude $(p)$ and response amplitude $(a)$.

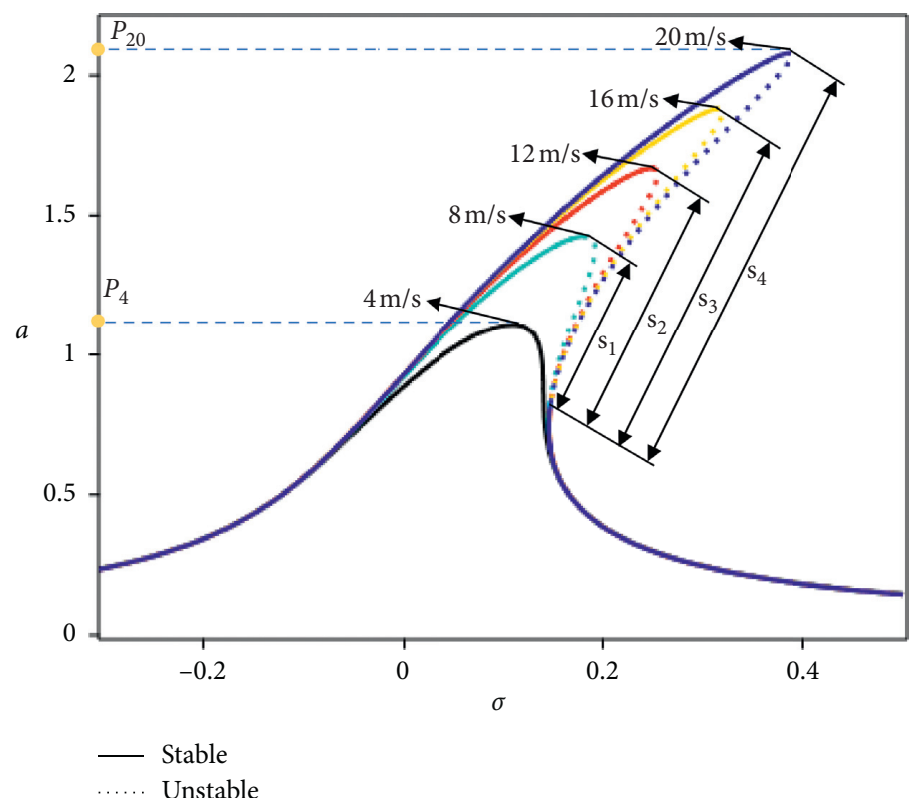

(a)

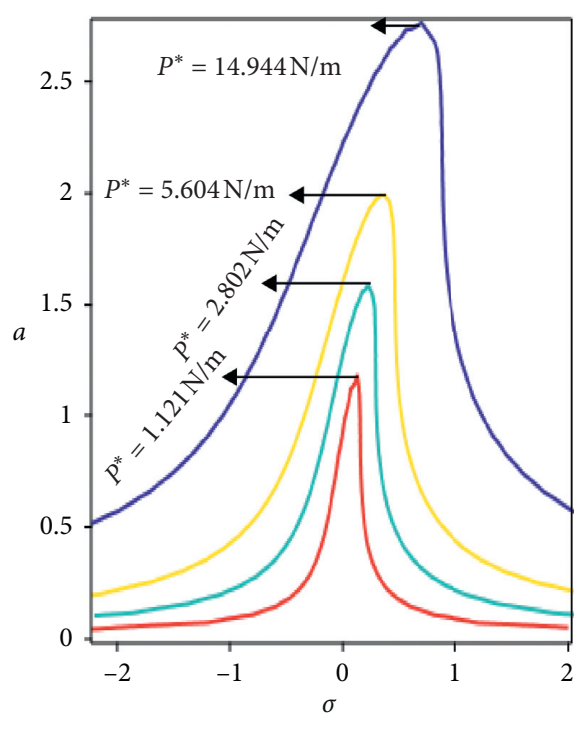

(b)

Figure 4: The frequency-amplitude curve of principal resonance. (a) Different velocities. (b) Different excitation amplitudes.

and the unit of excitation amplitude $\left(p^{*}\right)$ is $\mathrm{N} / \mathrm{m}$. It is worth noting that the excitation amplitude $(p)$ is obtained by substituting excitation amplitude $\left(p^{*}\right)$ into equations (17) and (18). And the definition of the unit of excitation amplitude $\left(p, p^{*}\right)$ and response amplitude $(a)$ in the figure is consistent with Figure 3, respectively. In addition, the 
excitation amplitude $(p=2,4,6,8$, and 10$)$ is substituted into equation (16), and the maximum positive amplitude of points $a_{1}, a_{2}, a_{3}, a_{4}$, and $a_{5}$ is obtained by using Runge-Kutta function in MATLAB [7].

As shown in Figure 3, the frequency-response curves are presented, so some numerical solutions could be provided in these curves to show the good agreements between the perturbation solutions and numerical ones. When the response amplitude is greater than 2, the error of the analytical solution will increase with the increase in excitation amplitude. In Figure 3, when the response amplitude is greater than 2, the error between the numerical solution and the analytic solution increases from $2.98 \%$ for $p=6$ to $5.42 \%$ for $p=10$. However, the frequency-amplitude curve can still be used for analysis.

In order to analyze the influence of wind velocity, excitation amplitude, and tension on the principal resonance of the forced self-excited system of iced transmission lines, the frequency-amplitude curve of equation (28) is drawn by Maple as shown in Figures 4-7. In the forced self-excited system, the change of excitation frequency always leads to complex resonance. Then, let the excitation frequency $\Omega=\omega$ in Figures 4-7. As the response amplitudes in equation (28) are in the form of $a^{2}$ or the higher power of $a$, the response amplitude $(a)$ of the frequency-amplitude curve is axisymmetric with respect to tuning parameters $(\sigma)$. In order to show the change of response amplitude intuitively, only the areas $(a>0)$ are taken for analysis, and the frequencyamplitude curve in this paper also takes the areas $(a>0)$. In Figure 4 and Figures 6-12, the steady-state solution and unsteady-state solution are studied. If the partial derivative $(\partial W(\sigma, a, p) / \partial a)$ of the curve is positive, it means that the curve is stable; if the partial derivative $(\partial W(\sigma, a, p) / \partial a)$ of the curve is negative, it means that the curve is unstable. And the solid lines indicate the stable steady-state solution, and dashed lines indicate the unstable steady-state solution [20].

Figure 4(a) shows the characteristics of response amplitude of principal resonance under different wind velocities with the excitation amplitude $p^{*}=0.187 \mathrm{~N} / \mathrm{m}$. With the increase in wind velocity, the frequency-amplitude curve of principal resonance shifts to the right, showing an obvious hardening behavior; and there are phenomena of multivalue and jump; the peak value of response amplitude increases from $P_{4}$ at $U=4 \mathrm{~m} / \mathrm{s}$ to $P_{20}$ at $U=20 \mathrm{~m} / \mathrm{s}$; the instability intervals of the frequency-amplitude curve also increase gradually, from interval $S_{1}$ with $U=8 \mathrm{~m} / \mathrm{s}$ to intervals $S_{2}, S_{3}$, and $S_{4}$ with $U=12,16$, and $20 \mathrm{~m} / \mathrm{s}$, respectively. It can be seen from Figure 4(a) that, with the increase in wind velocity, the resonance region becomes wider, and the region of multivalue of the curve increases; in a given tuning parameter region, the number of steady-state solutions increases from one to three. The multivalue of the response curve due to the nonlinearity is of significance from the physical point of view because it leads to jump phenomena. These results indicate that the behavior of iced transmission lines with increasing wind velocity is significantly nonlinear.

Besides the wind velocity, the excitation amplitude also has a great influence on the response amplitude. Figure 4(b) shows the effect of the excitation amplitude on the frequency-amplitude curve of principal resonance with wind velocity $U=4 \mathrm{~m} / \mathrm{s}$. The resonance curve tends to expand, and the peak value of response amplitude increases as the excitation amplitude increases.

In order to analyze the influence of tension and sag on the principal resonance of the forced self-excited system under the same line parameters and the same aerodynamic load, Figure 5 provides the frequency-amplitude curve under different tensions $\left(p^{*}=0.934 \mathrm{~N} / \mathrm{m} ; U=4 \mathrm{~m} / \mathrm{s}\right)$. According to the relation $\left(d=\left(\mathrm{mgl}^{2} / 8 \mathrm{H}\right)\right)$ between sag and tension [25], the frequencyamplitude curves with tension $H=15,000-70,000 \mathrm{~N}$ in Figure 5 are obtained by Maple, respectively. As shown in Figure 5(a), as the tension increases from $15,000 \mathrm{~N}$ to $30,000 \mathrm{~N}$, the frequencyamplitude curve is extended, and the peak value of response amplitude increases. However, as shown in Figure 5(b), as the tension increases from $30,000 \mathrm{~N}$ to $70,000 \mathrm{~N}$, the frequencyamplitude curve appears to shrink inward, and the peak value of response amplitude decreases.

When the tension $H$ changes from $15,000 \mathrm{~N}$ to $70,000 \mathrm{~N}$, the sag $d$ decreases, and the structural stiffness of the transmission line system increases. However, the response amplitude of the forced self-excited system increases first and then decreases. Therefore, in engineering applications, the galloping amplitude of iced transmission lines will reduce effectively by changing the tension appropriately.

In order to figure out the influence of Young's modulus on the principal resonance of the forced self-excited system under the same line parameters and the same aerodynamic load, Figure 6 analyzes the nonlinear characteristics of response amplitude under different Young's moduli with $p^{*}=$ $0.934 \mathrm{~N} / \mathrm{m}$ and $U=4 \mathrm{~m} / \mathrm{s}$.

As shown in Figure 6(a), as Young's modulus increases from $9560.7 \mathrm{~N} / \mathrm{mm}^{2}$ to $2,390,169 \mathrm{~N} / \mathrm{mm}^{2}$, the peak value of the frequency-amplitude curve of principal resonance decreases, and the resonant regions are obviously reduced. When Young's modulus is in this range $E=239,016.5-478,033 \mathrm{~N} /$ $\mathrm{mm}^{2}$, the frequency-amplitude curve of principal resonance shifts to the right, showing the nonlinear characteristics of hardening behavior, and there are phenomena of multivalue and jump.

As shown in Figure 6, as the tension increases from $20,000 \mathrm{~N}$ to $50,000 \mathrm{~N}$, the peak value of the frequency-amplitude curve decreases. And with the increase in tension and response amplitude, the possibility of multivalue and jump phenomena increases. To this end, in engineering applications, the galloping amplitude of iced transmission lines will reduce by increasing Young's modulus of iced transmission lines. Apart from this, the galloping amplitude of iced transmission lines can be effectively reduced by increasing the tension and Young's modulus at the same time.

Figure 7 (a) compares the nonlinear vibration characteristics of response amplitude of the principal resonance under different wind velocities, in which the tuning parameter $\sigma$ is 0.5 . When wind velocity $U$ is $8 \mathrm{~m} / \mathrm{s}$, the response amplitude has the phenomenon of multivalue. With the increase in wind velocity, the $p$ - $a$ curve of principal resonance shifts to the left, and the instability intervals of the frequency-amplitude curve also increase gradually. It can be seen from Figure 7 (a) that the region of multivalue of the 


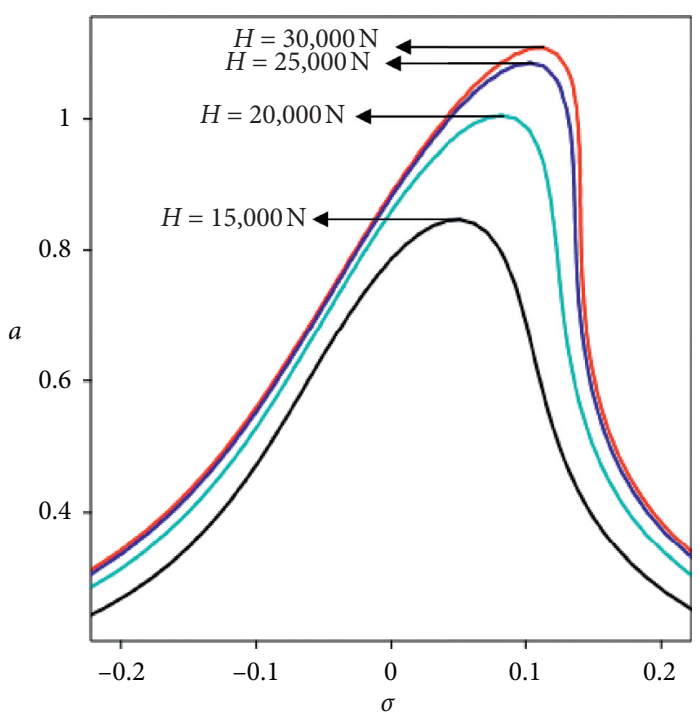

(a)

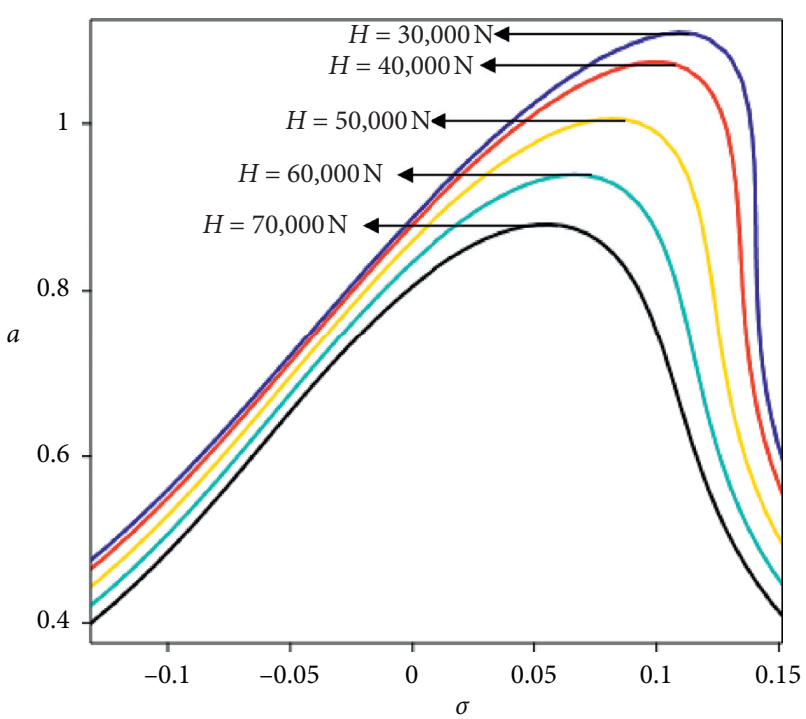

(b)

Figure 5: The frequency-amplitude curve of principal resonance. $(30,000-70,000 \mathrm{~N})$.

curve increases with the increase in wind velocity, and the number of steady-state solutions increases from one to three in a given excitation amplitude region. Moreover, with the increase in wind velocity, it is easier to generate the jump phenomenon of response amplitude with a small excitation amplitude $(p)$.

Figure 7(b) compares the characteristics of response amplitude of the principal resonance under different wind velocities and different tuning parameters. Similar with Figure $7(\mathrm{a})$, with the increase in wind velocity, the region of multivalue of the curve in Figure 7(b) increases; the number of steady-state solutions increases from one to three in a given excitation amplitude region. However, the larger the resonance amplitude is, the faster the instability intervals of the trivial solution increase under the influence of tuning parameters.

The wind velocity, excitation amplitude, tension, and Young's modulus have a great influence on the principal resonance of the forced self-excited system of iced transmission lines and even determine the conditions of the galloping of iced transmission lines.

\section{Forced Vibration Away from Principal Resonance}

Equation (20) is the forced vibration close to principal resonance, so it is generally considered that the excitation amplitude of the forced term $(p \cos (\Omega t))$ is small. However, for the forced vibration far away from resonance, it is considered that the excitation amplitude of the forced term $(p \cos (\Omega t))$ is large. Therefore, the solution form of small parameter $(\varepsilon)$ of the nonlinear galloping equation can be assumed as follows [30]:

$$
\begin{aligned}
q & =\varepsilon \bar{q}, \\
\mu^{*} & =\varepsilon \bar{\mu}, \\
\dot{q} & =\varepsilon \overline{\dot{q}}, \\
\dot{q}^{3} & =\varepsilon \dot{\dot{q}} .
\end{aligned}
$$

Substituting equation (29) into equation (16), it can be obtained that

$$
\ddot{q}+w^{2} q+\varepsilon\left[c_{1} q^{2}+c_{2} q^{3}+\left(\mu^{*}-c_{3}\right) \dot{q}+c_{4} \dot{q}^{3}\right]=p \cos (\Omega t) .
$$

The multiple-scale method has been discussed, and $q$, the partial differential operator symbol, and the frequency of external excitation are

$$
\begin{aligned}
q & =q_{0}+\varepsilon q_{1}, \\
D T & =D_{0} T_{0}+\varepsilon\left(D_{0} T_{1}+D_{1} T_{0}\right), \\
\Omega t & =\xi T_{0}+\varepsilon \sigma T_{1} .
\end{aligned}
$$

Substituting equation (31) into equation (30) and equating coefficients of like powers of $\varepsilon^{n}(n=0,1)$ led to the following linear ordinary equations, respectively:

$$
\begin{aligned}
D_{0}^{2} q_{0}+\omega^{2} q_{0}= & p \cos \left(\xi T_{0}+\varepsilon \sigma T_{1}\right) \\
D_{0}^{2} q_{1}+\omega^{2} q_{1}= & -\left[2 D_{0} D_{1} q_{0}+c_{1} q_{0}^{2}+c_{2} q_{0}^{3}+\left(\mu+c_{3}\right) D_{0} q_{0}\right. \\
& \left.+c_{4}\left(D_{0} q_{0}\right)^{3}\right] .
\end{aligned}
$$

In equations (32a) and (32b), $D_{\mathrm{k}}$ is the partial derivative of $T_{\mathrm{k}}$, and the solution of equation (32a) is

$$
q_{0}=A\left(T_{1}\right) e^{i \omega T_{0}}+\bar{A}\left(T_{1}\right) e^{-i \omega T_{0}}+B e^{i \Omega T_{0}}+\bar{B} e^{-i \Omega T_{0}},
$$




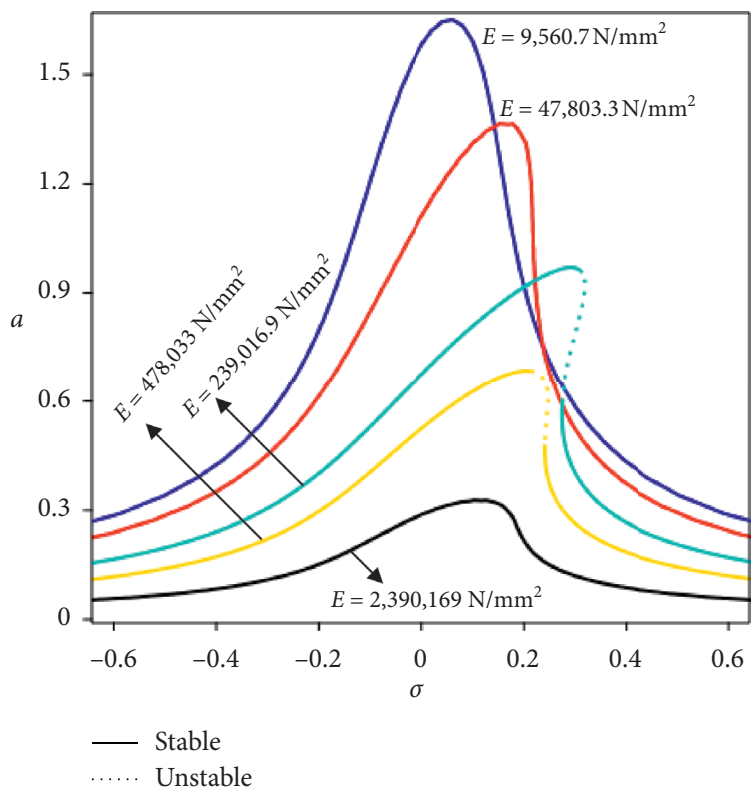

(a)

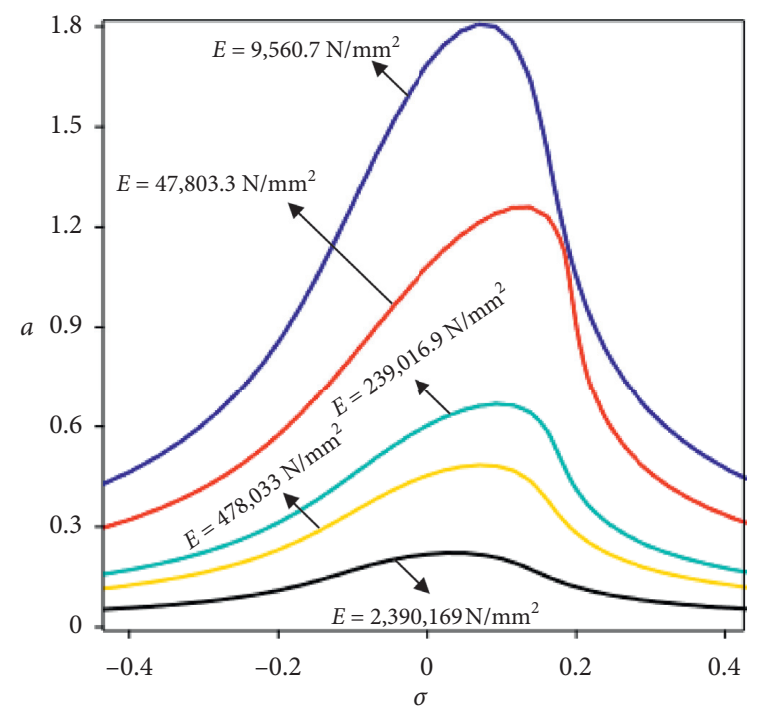

(b)

Figure 6: Continued. 


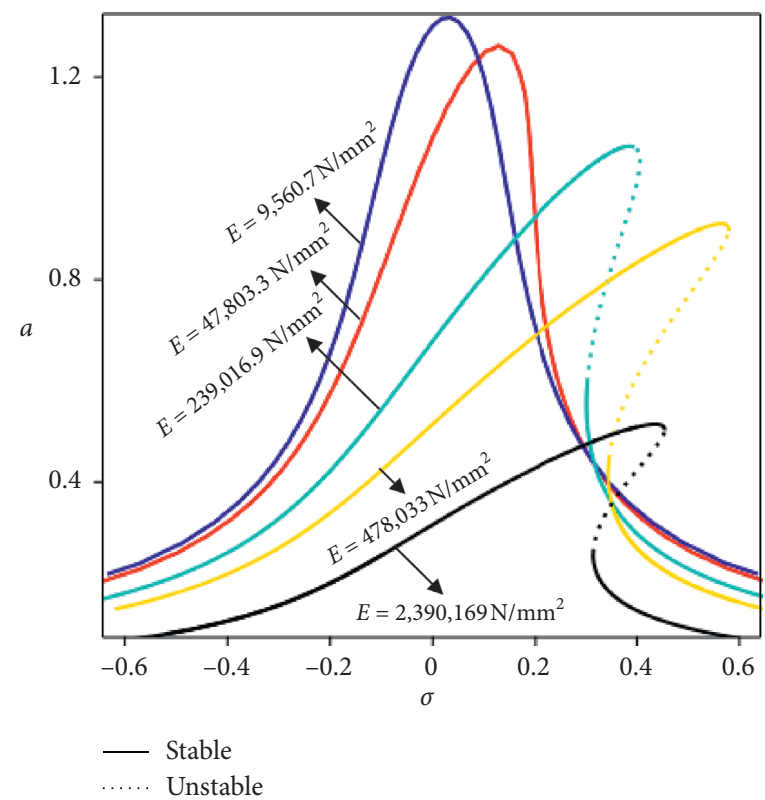

(c)

Figure 6: The frequency-amplitude curve of principal resonance. (a) Different Young's modulus (30,000 N). (b) Different Young's modulus $(20,000 \mathrm{~N})$. (c) Different Young's modulus $(50,000 \mathrm{~N})$.

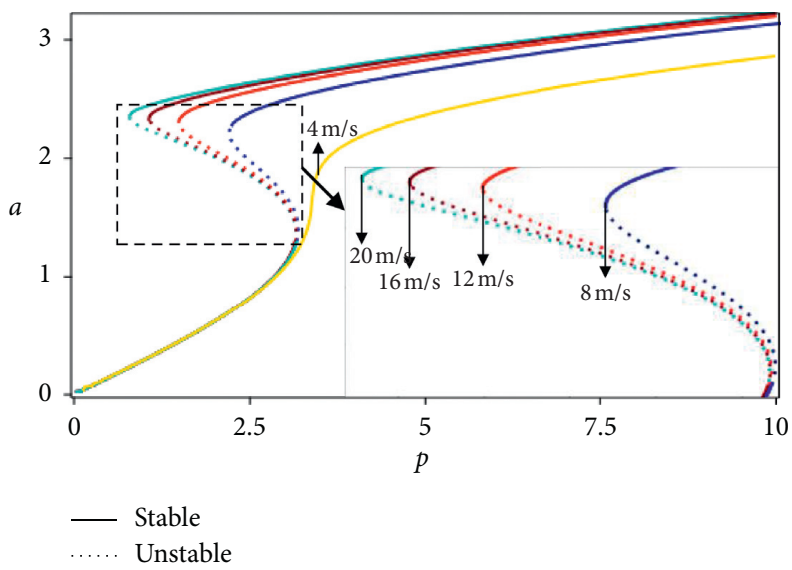

(a)

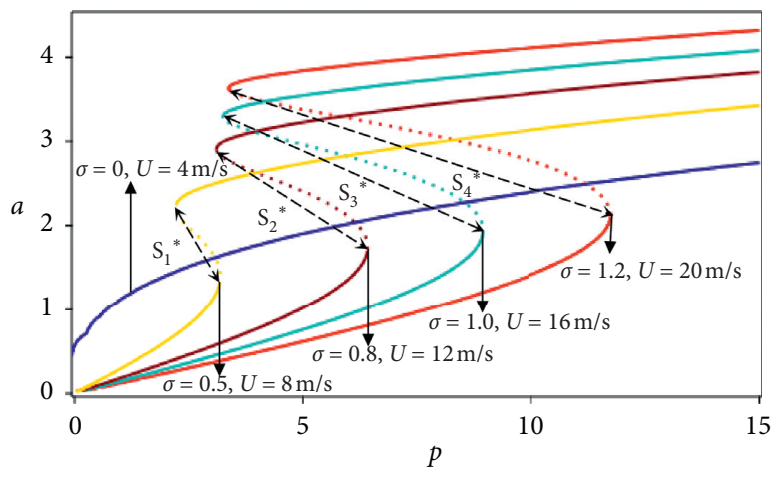

Stable

Unstable

(b)

Figure 7: The curves of excitation amplitude $(p)$ and response amplitude (a). (a) Different wind velocities $(\sigma=0.5)$. (b) Different wind velocities and tuning parameters. 
where $A\left(T_{1}\right)$ and $B\left(T_{1}\right)$ can be defined as

$$
\begin{aligned}
A\left(T_{1}\right) & =\frac{1}{2 a\left(T_{1}\right) \exp \left[i \beta\left(T_{1}\right)\right]}, \\
B & =\frac{P}{2\left|\omega^{2}-\Omega^{2}\right|} .
\end{aligned}
$$

Substituting equation (33) into equation (32b), it can be obtained that

$$
\begin{aligned}
& D_{0}^{2} q_{1}+\omega^{2} q_{1}=-2 i \omega D_{1}\left(A e^{i \omega t}+B e^{i \Omega t}\right)-c_{1}\left[A^{2} e^{2 i \omega t}+A \bar{A}+B \bar{B}+2 A B e^{2 i(\omega+\Omega) t}+2 i \bar{A} B e^{2 i(\Omega-\omega) t}+B^{2} e^{2 i \Omega t}\right]
\end{aligned}
$$

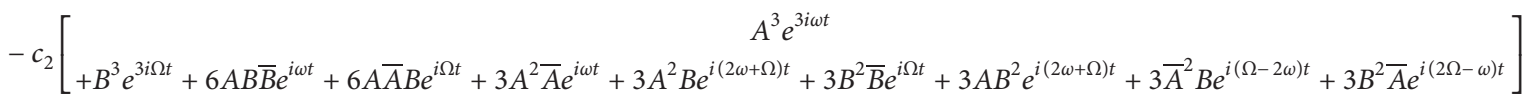

$$
\begin{aligned}
& -\left(\mu+c_{3}\right)\left(i \omega A e^{i \omega t}+i \Omega B e^{i \Omega t}\right) \\
& -c_{4}\left[\begin{array}{c}
6 i \omega^{2} A \bar{A} B e^{i \Omega t}-i \omega^{3} A^{3} e^{3 i \omega t}-i \Omega^{3} B^{3} e^{3 i \Omega t}+3 i \omega^{3} A^{2} \bar{A} e^{i \omega t}+6 i \omega \Omega^{2} A B \bar{B} e^{i \omega t} \\
+3 i \Omega^{3} B^{2} \bar{B} e^{i \Omega t}-3 i \Omega \omega^{2} A^{2} B e^{i(2 \omega+\Omega) t}-3 i \omega \Omega^{2} B^{2} A e^{i(2 \Omega+\omega) t}-3 i \omega \Omega^{2} A \bar{B}^{2} e^{i(\omega-2 \Omega) t}-3 i \Omega \omega^{2} B \bar{A}^{2} e^{i(\Omega-2 \omega) t}
\end{array}\right]+c c .
\end{aligned}
$$

According to equation (35), there are subharmonics and super harmonics in this system. When the excitation frequency is close to integer multiples and fractional multiples of the natural frequency $(\Omega=2 \omega+\varepsilon \sigma ; \Omega=3 \omega+\varepsilon \sigma ; \Omega=$ $(\omega / 2)+\varepsilon \sigma ; \Omega=(\omega / 3)+\varepsilon \sigma)$, the superharmonic resonance and the subharmonic resonance of the system of the transmission line would appear. In addition, the 1/2-order and 1/3-order subharmonic or 2-order and 3-order superharmonic resonances also belong to secondary resonance [31].

4.1.1/2-Order Subharmonic Resonance. When the excitation frequency $(\Omega)$ of the system is defined as 2 times of the natural frequency $(\omega)$, the system will produce the $1 / 2$-order subharmonic resonance. Let $\Omega=2 \omega+\varepsilon \sigma$ in equation (35); the averaging equation of response amplitude and phase can be obtained:

$$
\dot{a}=\frac{-\left(\mu+c_{3}\right)}{2} a-\frac{3}{8} \omega^{2} c_{4} a^{3}-\frac{c_{4} p^{2}}{3 \omega^{2}} a-\frac{p c_{1}}{6 \omega^{3}} a \cdot \sin \left(\sigma T_{1}-\beta\right),
$$

$a \dot{\beta}=\frac{3}{8 \omega} c_{2} a^{3}+\frac{c_{2} p^{2}}{12 \omega^{5}} a+\frac{p c_{1}}{6 \omega^{3}} a \cdot \cos \left(\sigma T_{1}-\beta\right)$.

Introduce $\gamma=\sigma T_{1}-\beta$ to acquire the steady-state solution of the amplitude and phase of equations (36a) and (36b), let $D_{1} A=0$, and obtain the frequency-amplitude equation by eliminating $\gamma$ :

$$
\left(\frac{p}{6 \omega^{3}} c_{1} a\right)^{2}=\left(\frac{\mu+c_{3}}{2} a+\frac{3}{8} \omega^{2} c_{4} a^{3}+\frac{p^{2}}{3 \omega^{2}} c_{4} a\right)^{2}+\left(\sigma a-\frac{3 c_{2}}{8 \omega} a^{3}-\frac{c_{2} p^{2}}{12 \omega^{5}} a\right)^{2} .
$$

In order to analyze the influence of wind velocity, excitation amplitude, and tension on the 1/2-order subharmonic resonance of the forced self-excited system of iced transmission lines, the frequency-amplitude curve of equation (37) is drawn by Maple in Figures 8 and 9.

Figure 8 discusses the characteristics of response amplitude under different excitation amplitudes, in which the wind velocity $U=4 \mathrm{~m} / \mathrm{s}$ and tension $H=30,000 \mathrm{~N}$. In Figure $8(\mathrm{a})$, when the excitation amplitude $p^{*}=0.747 \mathrm{~N} /$ $\mathrm{mm}^{2}$, the response amplitude $(a)$ of the $1 / 2$-order subharmonic resonance would be $0.400 \mathrm{~m}$. With the increase in excitation amplitude, the peak value of response amplitude will increase. In Figure 8(b), when the excitation amplitude $p^{*}>46.700 \mathrm{~N} / \mathrm{mm}^{2}$, the response amplitude (a) of the $1 / 2$-order subharmonic resonance will decrease with the increase in excitation amplitude, and the resonance region and the instability intervals of the curve also decrease and even disappear. Besides, with the increase in the excitation amplitude, the tuning parameters corresponding to the resonance peak and unstable point move to the positive direction. The peak value of response amplitude shifts to the positive direction continuously with the increase in excitation amplitude, which indicates that the 1/2-order subharmonic resonance of the forced self-excited system presents the characteristics of hardening behavior. However, with the increase in excitation amplitude, the hardening phenomenon of the system first increases and then decreases gradually.

In order to analyze the influence of tension, wind velocity, and tuning parameter on the 1/2-order subharmonic resonance of the forced self-excited system of ice-coated transmission lines, Figures 9(a)-9(d) compare the 


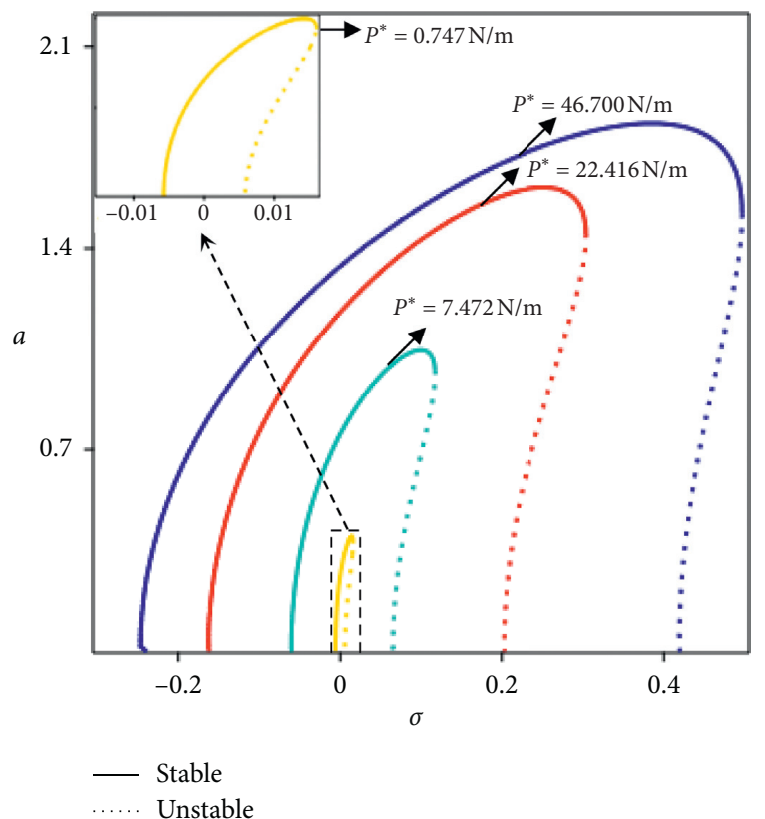

(a)

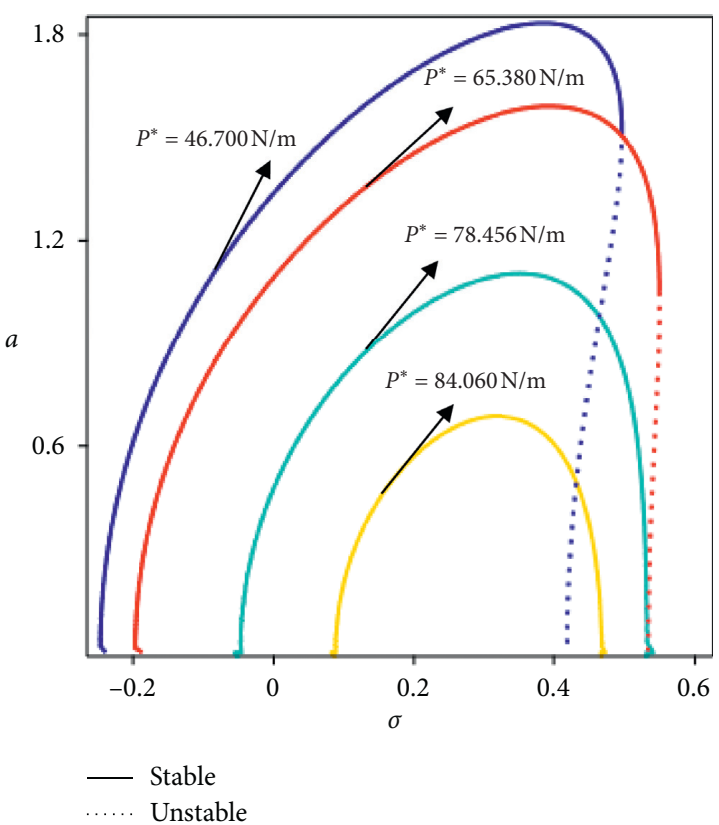

(b)

Figure 8: The frequency-amplitude curve of the 1/2-order subharmonic resonance. (a) Different excitation amplitudes $(0.747-46.700 \mathrm{~N} / \mathrm{m})$. (b) Different excitation amplitudes $(46.700-84.060 \mathrm{~N} / \mathrm{m})$.

characteristics of response amplitude of the 1/2-order subharmonic resonance under different tensions, different wind velocities, and different tuning parameters, respectively. And Young's modulus $E=47,803.3 \mathrm{~N} / \mathrm{mm}^{2}$ in Figure 9, the tension $H=30,000 \mathrm{~N}$ in Figures $9(\mathrm{~b})-9(\mathrm{~d})$, the tuning parameter $\sigma=0$ in Figures 9(a) and 9(b), and the wind velocity $U=4 \mathrm{~m} / \mathrm{s}$ in Figures 9 (a) and $9(\mathrm{c})$.

As shown in Figure 9(a), as the tension increases from $15,000 \mathrm{~N}$ to $70,000 \mathrm{~N}$, the peak value of response amplitude of the 1/2-order subharmonic resonance decreases continuously; the range of excitation amplitude $\left(p^{*}\right)$ corresponding to resonance increases first and then decreases.

As shown in Figure 9(b), as the wind velocity increases from $4 \mathrm{~m} / \mathrm{s}$ to $20 \mathrm{~m} / \mathrm{s}$, the response amplitude of the $1 / 2$ order subharmonic resonance increases continuously; however, the response amplitude increases more and more slowly with the increase in wind velocity; and the excitation amplitude $\left(p^{*}\right)$ range corresponding to the resonance region of the response amplitude (a) also expands.

As shown in Figure 9(c), the peak value of response amplitude increases first and then decreases with the increase in tuning parameter. When the tuning parameter $\sigma=0.07$, with the excitation amplitude increasing from 0 , the resonance amplitude is 0 at the beginning, which means there is no nontrivial solution; when the excitation amplitude increases to a certain value, the first bifurcation point appears, and on the right side after the bifurcation point, there is a nontrivial resonance solution, which is unstable; with the increase in tuning parameters, the excitation amplitude corresponding to the bifurcation point of the curve increases, that is, it means that the resonance peak and the tuning parameters corresponding to the unstable point move on the transverse axis; and the peak value of the response amplitude increases at first and then decreases.

Figure 9(d) compares the response amplitude of the 1/2order subharmonic resonance under different wind velocities and different tuning parameters. Similarly, when the tuning parameter $\sigma=0.5$ and the wind velocity $U=8 \mathrm{~m} / \mathrm{s}$, the same phenomenon as Figure 9(c) appears here. And when the excitation amplitude continues to increase, the number of resonance solutions changes from two to one, and the resonance solutions after this point are stable. Moreover, with the increase in wind velocity and tuning parameters, the peak value of the response amplitude of the curve increases faster, and the resonance peak and the tuning parameters corresponding to the unstable point move on the abscissa axis. With the increase in wind velocity and tuning parameter at the same time, the region of instability of response amplitude increases continuously. And the peak value of response amplitude shifts to the positive direction continuously with the increase in excitation amplitude.

4.2. 2-Order Superharmonic Resonance. When the excitation frequency $(\omega)$ is defined as $1 / 2$ times of the natural frequency $(\Omega)$ of the system, the system will produce 2-order superharmonic resonance. Let $\Omega=(\omega / 2)+\varepsilon \sigma$ in equation (35); 


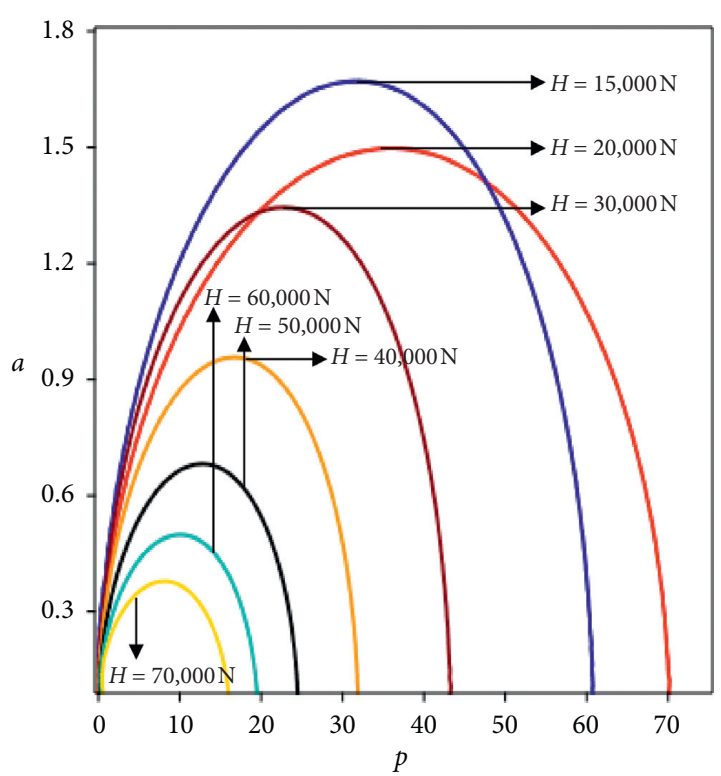

(a)

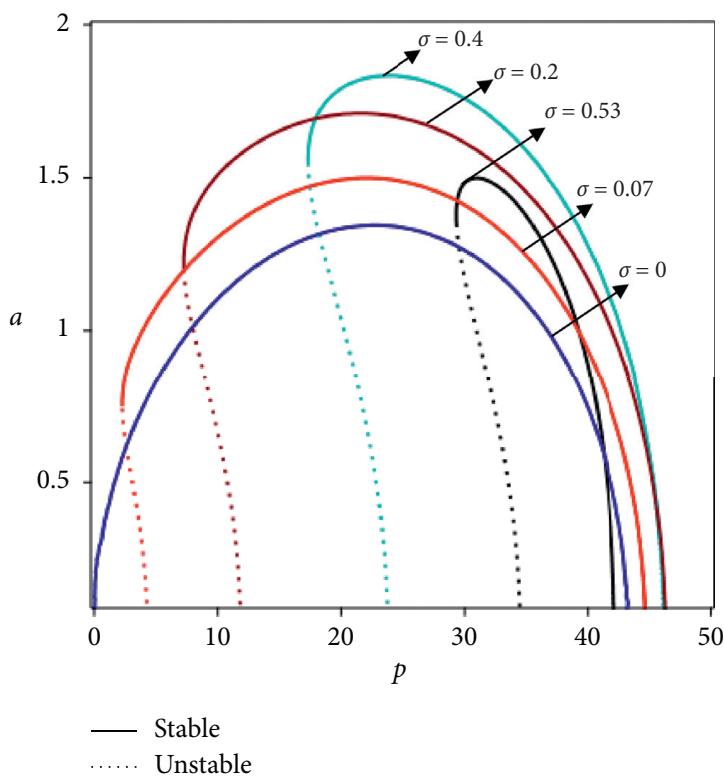

(c)

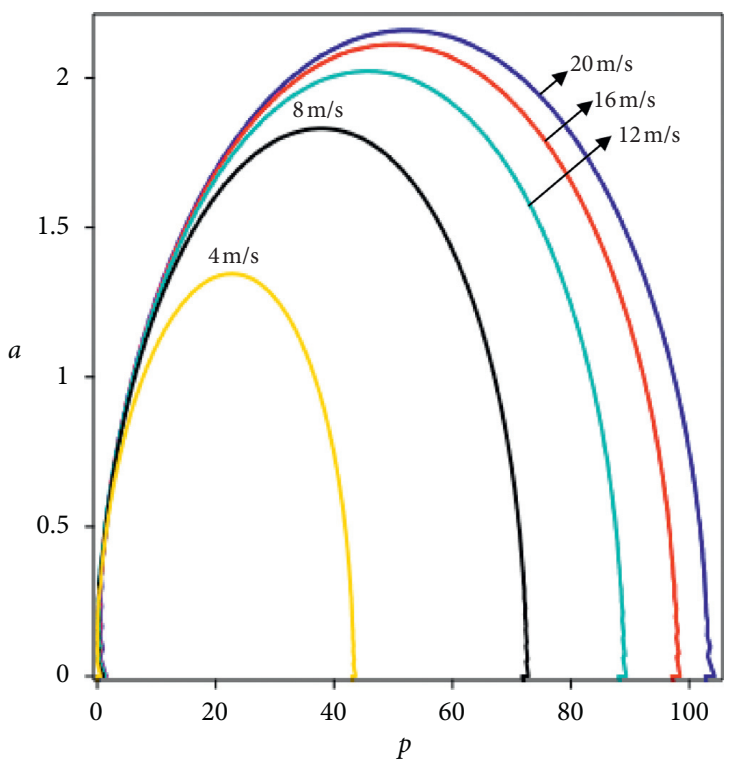

(b)

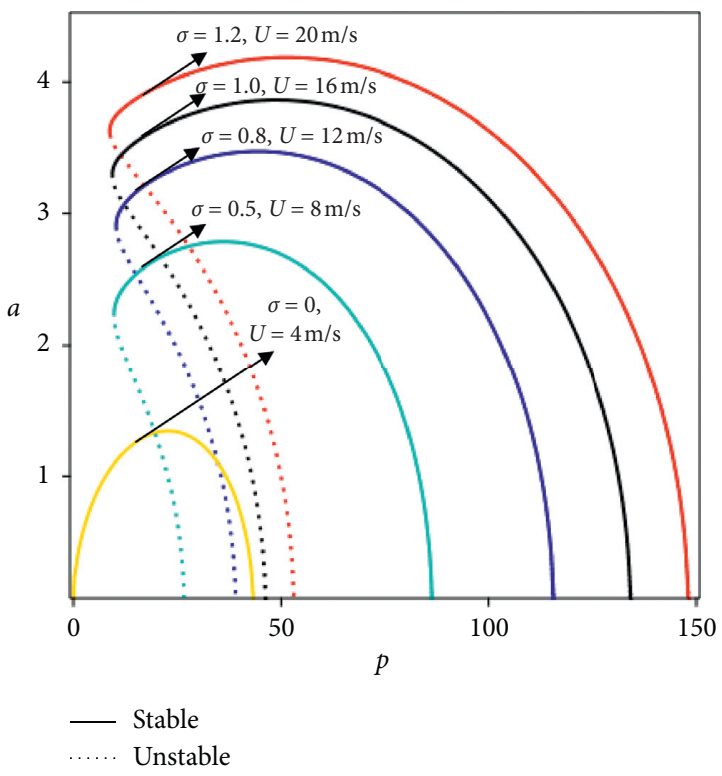

(d)

Figure 9: The curves of $(p)$ and (a) of 1/2 subharmonic resonance. (a) Different tensions. (b) Different wind velocities. (c) Different tuning parameters. (d) Different wind velocity and tuning parameters.

the averaging equation of response amplitude and phase can be obtained:

$$
\begin{aligned}
& \dot{a}=\frac{-\left(\mu+c_{3}\right)}{2} a-\frac{3}{8} \omega^{2} c_{4} a^{3}-\frac{c_{4} p^{2}}{3 \omega^{2}} a+\frac{4 c_{1} p^{2}}{3 \omega^{5}} \cdot \sin \left(\sigma T_{1}-\beta\right), \\
& \dot{a}=\frac{3}{8 \omega} c_{2} a^{3}+\frac{4 c_{2} p^{2}}{3 \omega^{5}} a+\frac{4 c_{1} p^{2}}{3 \omega^{5}} \cdot \cos \left(\sigma T_{1}-\beta\right) .
\end{aligned}
$$


Introduce $\gamma=\sigma T_{1}-\beta$ to acquire the steady-state solution of the amplitude and phase of equation (30), let
$D_{1} A=0$, and obtain the frequency-amplitude equation by eliminating $\gamma$ :

$$
\left(\frac{4 c_{1} p^{2}}{9 \omega^{5}}\right)^{2}=\left(\sigma a-\frac{3}{8 \omega} c_{2} a^{3}-\frac{4 c_{2} p^{2}}{3 \omega^{5}} a\right)^{2}+\left(\frac{\left(\mu+c_{3}\right)}{2} a+\frac{3}{8} \omega^{2} c_{4} a^{3}+\frac{c_{4} p^{2}}{3 \omega^{2}} a\right)^{2} .
$$

4.3.3-Order Superharmonic Resonance. When the excitation frequency $(\Omega)$ is defined as $1 / 3$ times of the natural frequency $(\omega)$ of the system, the system will produce 3 -order superharmonic resonance. Let $\Omega=(\omega / 3)+\varepsilon \sigma$ in equation (35); the averaging equation of response amplitude and phase can be obtained:

$$
\begin{aligned}
& \dot{a}=\frac{-\left(\mu+c_{3}\right)}{2} a-\frac{3}{8} \omega^{2} c_{4} a^{3}-\frac{27 p^{2}}{256 \omega^{2}} c_{4} a+\frac{c_{4}}{81} \cdot \frac{729 p^{3}}{4096 \omega^{4}} \cos \left(\sigma T_{1}-\beta\right)+3 c_{2} \cdot \frac{729 p^{3}}{4096 \omega^{7}} \sin \left(\sigma T_{1}-\beta\right), \\
& \dot{a}=\frac{3}{8 \omega} c_{2} a^{3}+\frac{243 c_{2} p^{2}}{256 \omega^{5}} a-\frac{c_{4}}{81} \cdot \frac{729 p^{3}}{4096 \omega^{4}} \sin \left(\sigma T_{1}-\beta\right)+3 c_{2} \cdot \frac{729 p^{3}}{4096 \omega^{7}} \cos \left(\sigma T_{1}-\beta\right) .
\end{aligned}
$$

Introduce $\gamma=\sigma T_{1}-\beta$ to acquire the steady-state solution of the amplitude and phase of equations (40a) and (40b), let $D_{1} A=0$, and obtain the frequency-amplitude equation by eliminating $\gamma$ :

$$
\left(\frac{81 c_{4} p^{3}}{4096 \omega^{4}}\right)^{2}+\left(\frac{2187 c_{2} p^{3}}{4096 \omega^{7}}\right)^{2}=\left(\frac{\mu+c_{3}}{2} a+\frac{3}{8} \omega^{2} c_{4} a^{3}+\frac{27 p^{2}}{256 \omega^{2}} c_{4} a\right)^{2}+\left(\sigma a-\frac{3}{8 \omega} c_{2} a^{3}-\frac{243 c_{2} p^{2}}{256 \omega^{5}} a\right)^{2}
$$

In order to analyze the influence of tension, excitation amplitude, tuning parameter, and wind velocity on the superharmonic resonance of the forced self-excited system of iced transmission lines, the frequency-amplitude curve of the superharmonic resonance is drawn by Maple as shown in Figures 10-12.

Figure 10 discusses the response amplitude of the superharmonic resonance under different excitation amplitudes with $U=4 \mathrm{~m} / \mathrm{s}, E=47,803.3 \mathrm{~N} / \mathrm{mm}^{2}$, and $H=30,000 \mathrm{~N}$. Figure $10(\mathrm{a})$ is obtained by substituting the excitation amplitude $p^{*}=1.868-22.416 \mathrm{~N} / \mathrm{mm}^{2}$ into equation (39). When the excitation amplitude $p^{*}=1.868 \mathrm{~N} / \mathrm{mm}^{2}$, the response amplitude $(a)$ of the 2 -order superharmonic resonance would be $0.430 \mathrm{~m}$. Figure $10(\mathrm{~b})$ is obtained by substituting the excitation amplitude $p^{*}=7.472-29.888 \mathrm{~N} / \mathrm{mm}^{2}$ into equation (41). When the excitation amplitude $p^{*}=7.472 \mathrm{~N} / \mathrm{mm}^{2}$, the response amplitude $(a)$ of the 3 -order superharmonic resonance would be $0.360 \mathrm{~m}$. In addition, the phenomenon of the 2 -order and the 3 -order superharmonic resonance is the same as the principal resonance. The peak value of response amplitude increases with the increase in excitation amplitude. Similarly, the peak value of response amplitude continuously shifts to the positive direction with the increase in excitation amplitude, showing the nonlinear characteristics of hardening behavior. And the resonance peak region of the superharmonic curve also continues to expand as the excitation amplitude increases.

Figure 11 is obtained by substituting different wind velocities, different tuning parameters, and different tensions into equation (39). And Young's modulus $E=47,803.3 \mathrm{~N} /$ $\mathrm{mm}^{2}$ in Figure 11, the wind velocity $U=4 \mathrm{~m} / \mathrm{s}$ in Figures 11(a) and 11(b), the tuning parameter $\sigma=0$ in Figure 11(a), and the tension $H=30,000 \mathrm{~N}$ in Figures 11(b) and 11(c).

As shown in Figure 11(a), as the excitation amplitude increases, the response amplitude increases gradually, and the response amplitude tends to a constant value. As the tension increases from $15,000 \mathrm{~N}$ to $70,000 \mathrm{~N}$, the response amplitude of the 2-order superharmonic resonance decreases continuously. Likewise, the peak value of response amplitude increases with the increase in tuning parameter in Figure 11(b). And the response amplitude tends to a constant value with the increase in excitation amplitude.

Figure 11(c) compares the nonlinear characteristics of response amplitude of the 2-order superharmonic resonance under different wind velocities and different tuning parameters. Similarly, with the increase in wind velocity and tuning parameter at the same time, the peak value of the response amplitude increases continuously. In addition, 


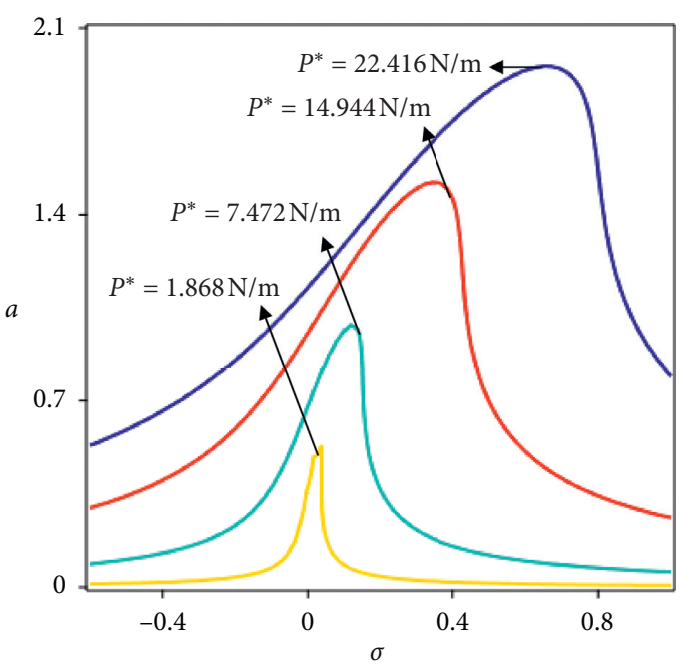

(a)

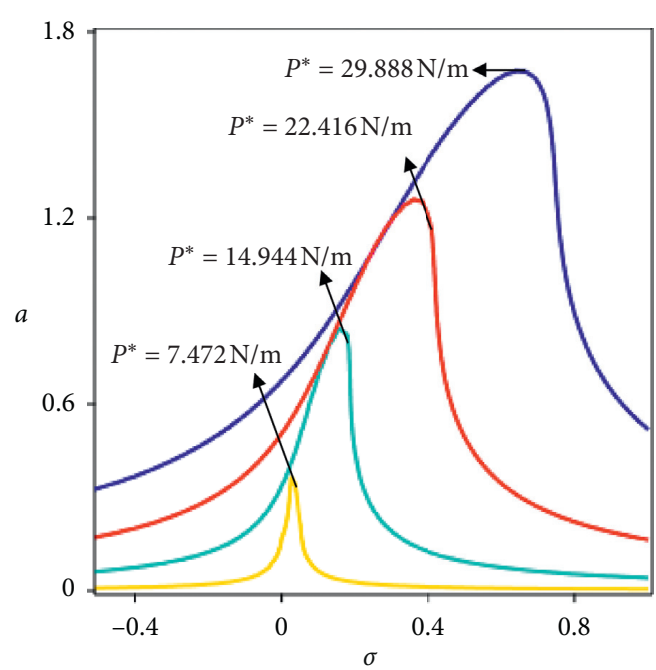

(b)

FIgURE 10: The frequency-amplitude curve of the superharmonic resonance. (a) The 2-order superharmonic resonance. (b) The 3-order superharmonic resonance.

with the increase in wind velocity and tuning parameter, the instability interval of the trivial solution also continues to increase.

Given Young's modulus $E=47,803.3 \mathrm{~N} / \mathrm{mm}^{2}$ in Figure 12 , the wind velocity $U=4 \mathrm{~m} / \mathrm{s}$ in Figures $12(\mathrm{a})-12(\mathrm{c})$, the tuning parameter $\sigma=0$ in Figures 12(a) and 12(b), and the tension $H=30,000 \mathrm{~N}$ in Figures $12(\mathrm{c})$ and $12(\mathrm{~d})$. And Figure 12 is obtained by substituting different tensions, different tuning parameters, and different wind velocities into equation (42).

As shown in Figure 12(a), as the excitation amplitude increases, the response amplitude of the 3-order superharmonic resonance increases gradually. When the tension $H=15,000-30,000 \mathrm{~N}$, the larger the tension is, the faster the response amplitude increases with the increase in excitation amplitude. As shown in Figure 12(b), with the increase in excitation amplitude, the response amplitude of 3-order superharmonic resonance also continues to increase. Additionally, with the tension increasing from $30,000 \mathrm{~N}$ to $70,000 \mathrm{~N}$, the phenomenon of right shift appears in Figure 12(b).
As shown in Figure 12(c), the peak value of response amplitude increases with the increase in tuning parameter. And the response amplitude continuously increases with the increase in excitation amplitude.

Also, Figure 12(d) compares the nonlinear characteristics of response amplitude of the 3-order superharmonic resonance under different wind velocities and different tuning parameters. Likewise, with the increase in wind velocity and tuning parameter at the same time, the peak value of the response amplitude continues to increase. Additionally, with the increase in wind velocity and tuning parameter, the instability intervals of the trivial solution also continue to increase.

4.4. 1/3-Order Subharmonic Resonance. When the natural frequency $(\omega)$ of the system is defined as 3 times of the excitation frequency $(\Omega)$, the system will produce $1 / 3$-order subharmonic resonance. Let $\Omega=3 \omega+\varepsilon \sigma$ in equation (35); the averaging equation of response amplitude and phase can be obtained:

$$
\begin{aligned}
& \dot{a}=\frac{-\left(\mu+c_{3}\right)}{2} a-\frac{3}{8} \omega^{2} c_{4} a^{3}-\frac{27 c_{4} p^{2}}{256 \omega^{2}} a-\frac{3 c_{2} p}{64 \omega^{3}} a^{2} \cdot \sin \left(\sigma T_{1}-\beta\right)+\frac{9 c_{4} p}{64} a^{2} \cdot \cos \left(\sigma T_{1}-\beta\right), \\
& \dot{a}=\frac{3}{8 \omega} c_{2} a^{3}+\frac{3 c_{2} p^{2}}{256 \omega^{5}} a+\frac{3 c_{2} p}{64 \omega^{3}} a^{2} \cdot \cos \left(\sigma T_{1}-\beta\right)+\frac{9 c_{4} p}{64} a^{2} \cdot \sin \left(\sigma T_{1}-\beta\right) .
\end{aligned}
$$

Introduce $\gamma=\sigma T_{1}-\beta$ to acquire the steady-state solution of the amplitude and phase of equations (42a) and (42b), let $D_{1} A=0$, and obtain the frequency-amplitude equation by eliminating $\gamma$ : 


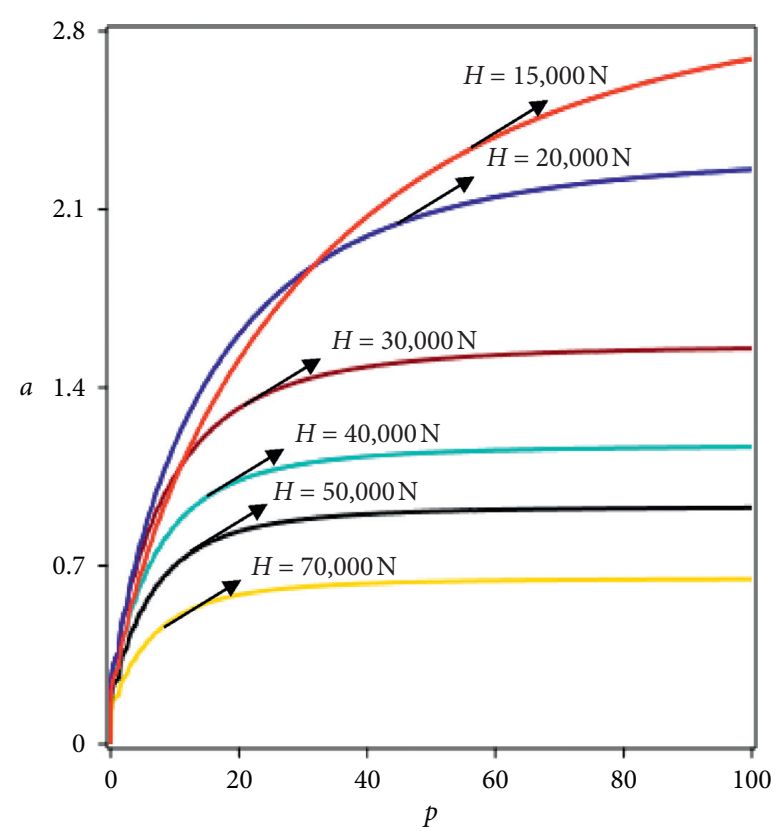

(a)

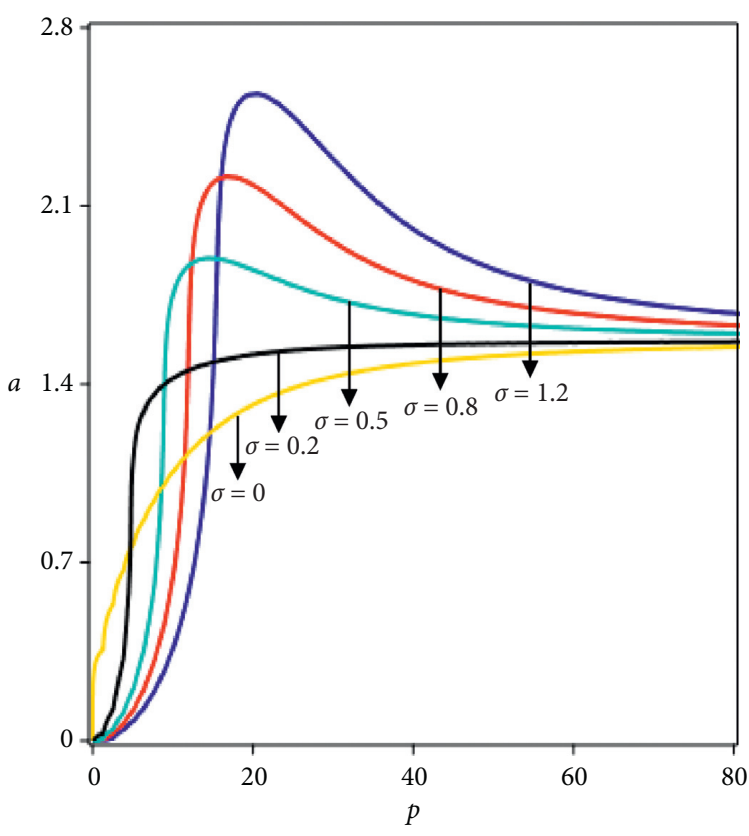

(b)

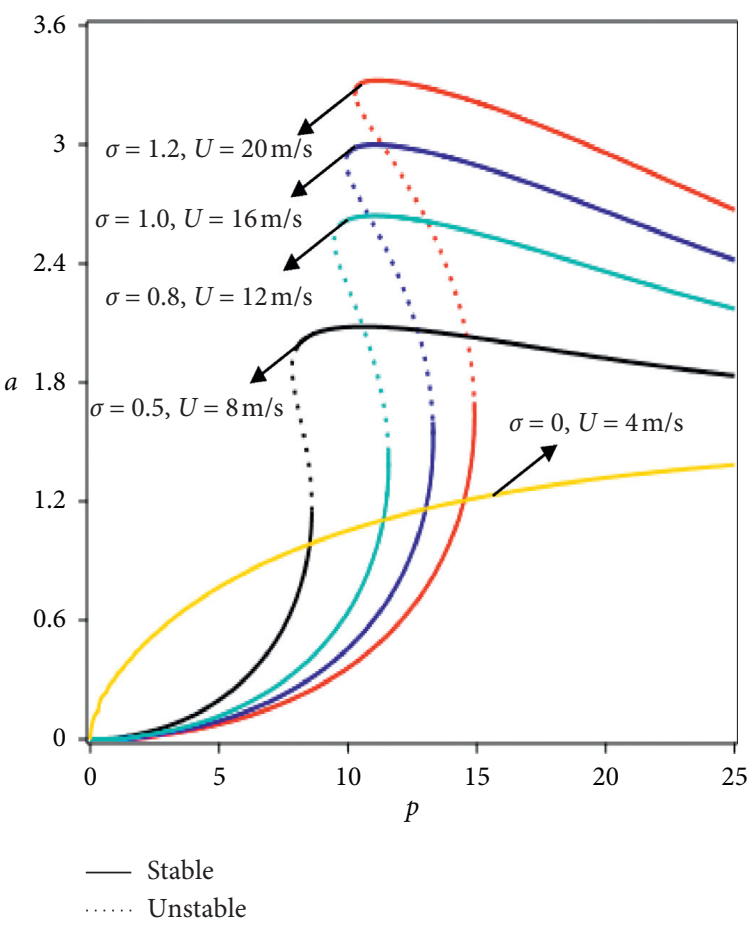

(c)

Figure 11: The curves of $(p)$ and (a) of 2-order superharmonic resonance. (a) Different tensions. (b) Different tuning parameters. (c) Different wind velocity and tuning parameters.

$$
\frac{9 p^{2} a^{4}}{4096} \cdot\left(9 c_{4}{ }^{2}+\frac{c_{2}{ }^{2}}{\omega^{6}}\right)=\left(\frac{\mu+c_{3}}{2} a+\frac{3}{8} \omega^{2} c_{4} a^{3}+\frac{27 c_{4} p^{2}}{256 \omega^{2}} a\right)^{2}+\left(\sigma a-\frac{3 c_{2}}{8 \omega} a^{3}-\frac{3 c_{2} p^{2}}{256 \omega^{5}} a\right)^{2}
$$

Given the condition of $U=4 \mathrm{~m} / \mathrm{s}, E=47,803.3 \mathrm{~N} / \mathrm{mm}^{2}$, and $H=30,000 \mathrm{~N}$, Figure 13 discusses the response amplitude of the 1/3-order subharmonic resonance under different excitation amplitudes. Figure 13 is obtained by substituting the excitation amplitude $p^{*}=54.172-112.080 \mathrm{~N} / \mathrm{mm}^{2}$ into equation (43). 


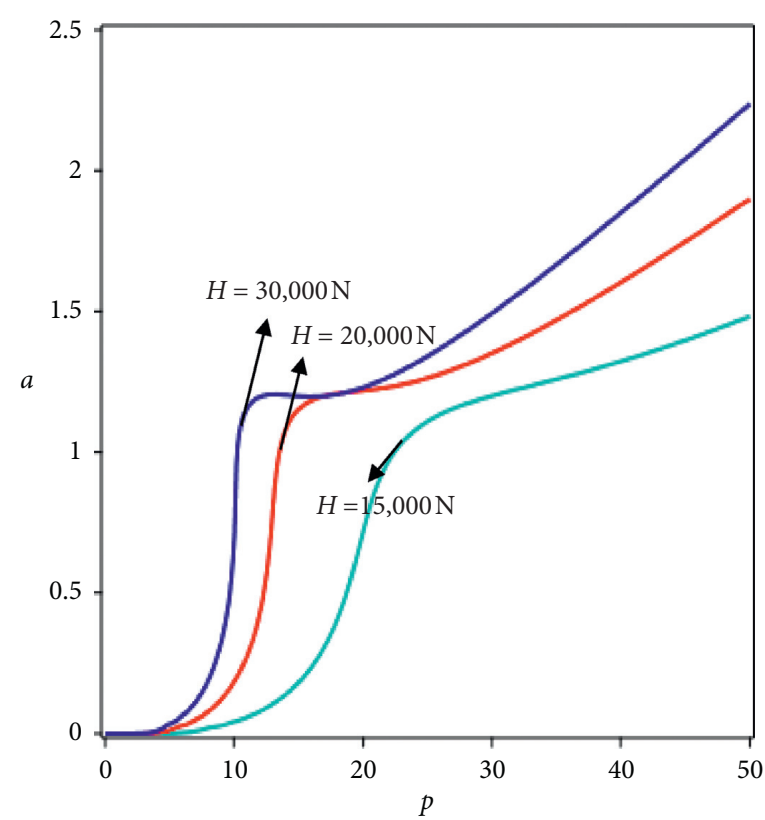

(a)

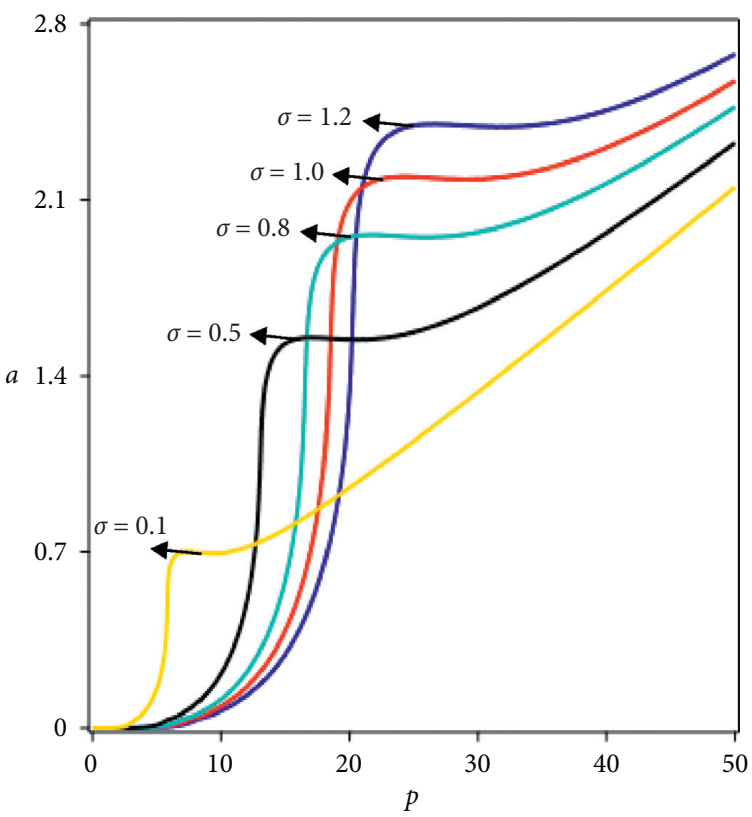

(c)

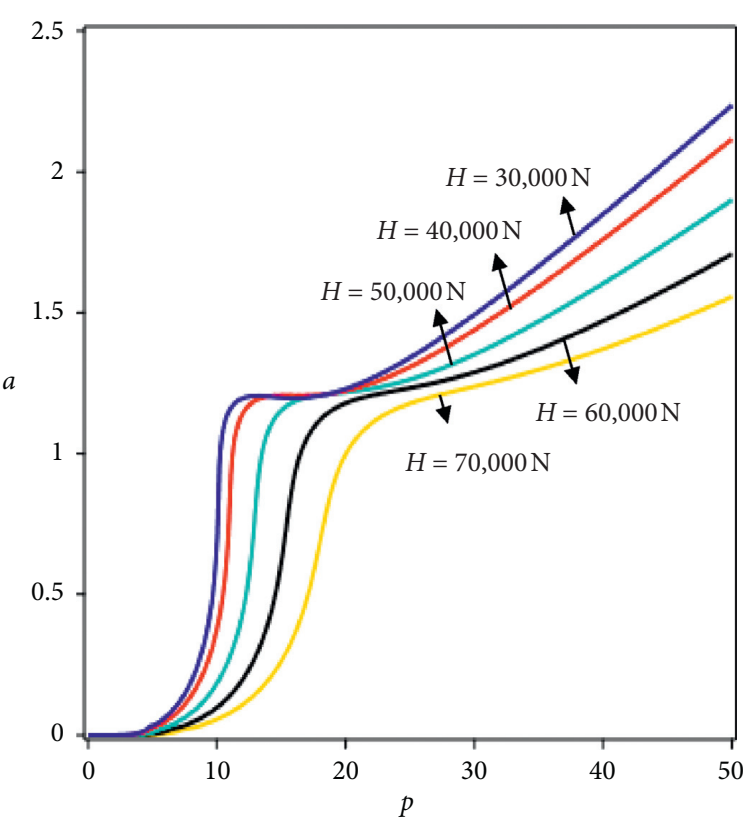

(b)

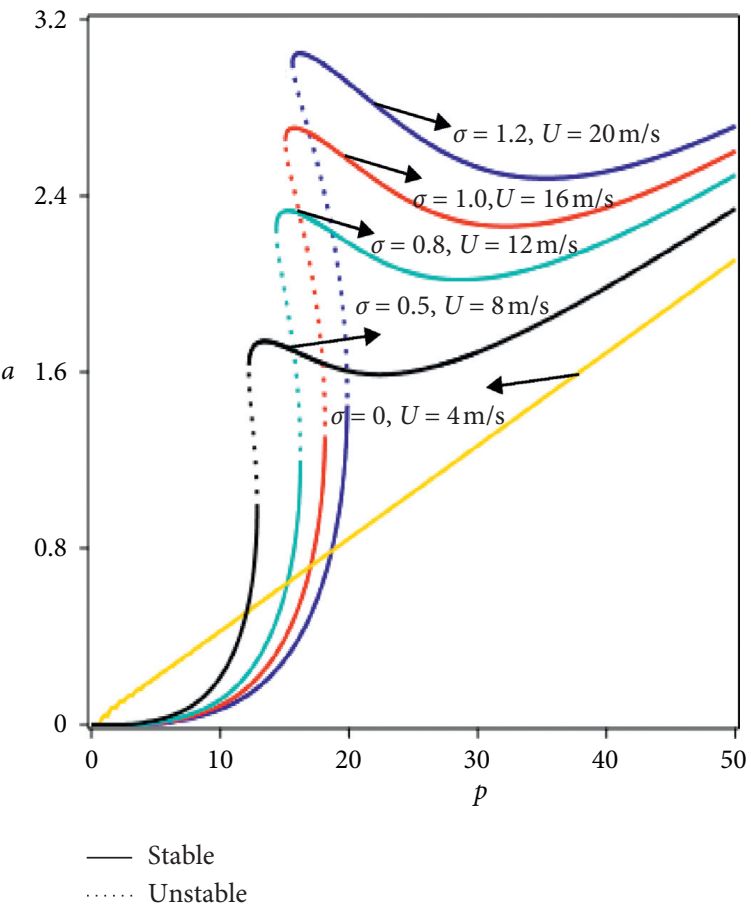

(d)

Figure 12: The curves of $(p)$ and (a) of 3-order superharmonic resonance. (a) Different tensions $((H)=15,000-30,000 \mathrm{~N})$. (b) Different tensions $((H)=30,000-70,000 \mathrm{~N})$. (c) Different tuning parameters. (d) Different wind velocity and tuning parameters.

As shown in Figure 13, the excitation amplitude of the 1/ 3-order subharmonic resonance is several times that of principal resonance. And it is very difficult to excite the $1 / 3$ order subharmonic resonance in the iced transmission line system. Therefore, the influence of the 1/3-order subharmonic resonance on iced transmission lines is small. As general research and design, it is not necessary to consider the 1/3-order subharmonic resonance. 


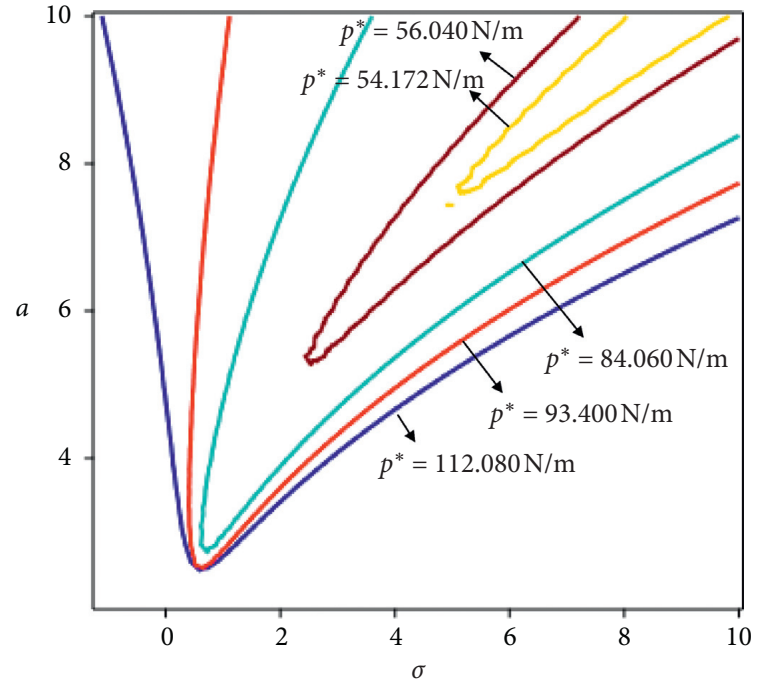

Figure 13: The frequency-amplitude curve of the 1/3-order subharmonic resonance.

\section{Conclusions}

A new forced self-excited system has been formulated to describe the influence of dynamic wind on the nonlinear galloping characteristics of iced transmission lines. The analytical solution of the nonlinear galloping equation is solved by the multiple-scale method. The principal resonance under weak excitation and the harmonic resonance under strong excitation are discussed, respectively:

(1) The change of control parameters (wind velocity, tension, excitation amplitude, Young's modulus, and tuning parameter) has a significant influence on the resonance peak, resonance region, and dynamic behavior of the principal resonance and the harmonic resonance. By changing these control parameters, the resonance region of the system can be changed, and the amplitude solution of the system can be transformed from a single value to multivalues so that the resonance phenomenon can be suppressed or excited.

(2) The principal resonance, 1/2-order subharmonic resonance, and 2-order and 3-order superharmonic resonances can affect galloping characteristics of iced transmission lines. Under the condition of harmonic excitation, the critical wind velocity needed for galloping of iced transmission line is lower, and the time needed for galloping of iced transmission line is shorter; and the peak value of the response amplitude of the transmission line increases, and the response amplitude also shows a jump phenomenon. The harmonic excitation reduces the service life of the transmission line, so the influence of principal resonance and harmonics should be considered in the structural parameter design. Designers can improve the conditions of the transmission line galloping and reduce the galloping amplitude by changing the structural parameters of the transmission line or taking measures to prevent galloping.
(3) The principal resonance of the forced self-excited system under weak excitation: as the wind velocity increases, the response amplitude of principal resonance will have the multivalue phenomenon, jump phenomenon, and hardening behavior; besides, the instability intervals of the trivial solution will expand. The harmonic resonance of the forced self-excited system under strong excitation: when the tuning parameter $(\sigma)$ is greater than 0 , the response amplitudes of the principal resonance, 2-order and 3order superharmonic resonances, and 1/2-order subharmonic resonance will have multivalue and jump phenomena; when the tuning parameter $(\sigma)$ is greater than 0 and the wind velocity increases, the instability intervals of the principal resonance will be faster, and harmonic resonance will also expand.

(4) The harmonic resonance of the forced self-excited system under strong excitation: when the excitation frequency is close to the integral and fractional times of the natural frequency, it is easier to produce the $1 /$ 2 -order subharmonic resonance, 2-order superharmonic resonance, and 3-order superharmonic resonance. As a result of the influence of the 1/3order subresonance on the transmission line is small, it is not necessary to consider the 1/3-order subharmonic resonance for the general research and design.

(5) The resonance peak value of the principal resonance decreases with the increase in Young's modulus. Moreover, with the increase in tension, the resonance peak value of the principal resonance increases first and then decreases. In engineering applications, increasing the tension and Young's modulus properly can reduce the resonance peak value of the principal resonance and harmonic resonance.

\section{Data Availability}

The authors declare that the data used to support the findings of this study are available from the corresponding author upon request.

\section{Conflicts of Interest}

The authors declare that there are no conflicts of interest regarding the publication of this paper.

\section{Acknowledgments}

This work was financially supported by the National Natural Science Foundation of China (51507106 and 51308570), Cutting Edge Project of Chongqing Science and Technology Commission (cstc2017jcyjAX0246), Graduate Research and Innovation Project of Chongqing (cys19240), China Postdoctoral Science Foundation (2720M672238), Chengdu International Science and Technology Cooperation Support Funding (2720GH02-00059-HZ), and Technology Research Project of Chongqing Education Commission (KJ201600712182). 


\section{References}

[1] B. Yan, X. Liu, X. Lv, and L. Zhou, "Investigation into galloping characteristics of iced quad bundle conductors," Journal of Vibration and Control, vol. 22, no. 4, pp. 968-987, 2016.

[2] M. Cai, L. Zhou, H. Lei, and H. Huang, "Wind tunnel test investigation on unsteady aerodynamic coefficients of iced 4bundle conductors," Advances in Civil Engineering, vol. 2019, Article ID 2586242, 12 pages, 2019.

[3] X. Liu, L. Liu, M. Cai, and B. Yan, "Free vibration of transmission lines with multiple insulator strings using refined models," Applied Mathematical Modelling, vol. 67, pp. 252-282, 2019.

[4] W. Lou, J. Wang, Y. Chen et al., "Effect of motion path of downburst on wind-induced conductor swing in transmission line," Wind and Structures, vol. 23, no. 3, pp. 41-59, 2016.

[5] B. Liu, K. Zhu, X. Li, and X. Zhan, "Hysteresis phenomenon in the galloping of the D-Shape iced conductor," Mathematical Problems in Engineering, vol. 2013, pp. 1-11, Article ID 784239, 2013.

[6] J. Den Hartog, "Transmission lines vibration due to sleet," Transactions of the American Institute of Electrical Engineers, vol. 51, no. 4, pp. 1074-1076, 1933.

[7] X. Liu, G. Min, C. Wu, and M. Cai, "Investigation on Influences of Two Discrete Methods on Galloping Characteristics of Iced Quad Bundle Conductors," Advances in Civil Engineering, vol. 2020, no. 1, pp. 1-17, Article ID 8818728, 2020.

[8] C. L. Lee and N. C. Perkins, "Experimental investigation of isolated and simultaneous internal resonances in suspended cables," Journal of Vibration and Acoustics, vol. 117, no. 4, pp. 385-391, 1995.

[9] J. Hu and P. F. Pai, "Experimental study of resonant vibrations of suspended steel cables using a 3D motion analysis system," Journal of Engineering Mechanics, vol. 138, no. 6, pp. 640-661, 2012.

[10] V. Gattulli, L. Martinelli, F. Perotti, and F. Vestroni, "Nonlinear oscillations of cables under harmonic loading using analytical and finite element models," Computer Methods in Applied Mechanics and Engineering, vol. 193, no. 1-2, pp. 69-85, 2004.

[11] S. R. K. Nielsen and P. H. Kirkegaard, "Super and combinatorial harmonic response of flexible elastic cables with small sag," Journal of Sound and Vibration, vol. 251, no. 1, pp. 79-102, 2002.

[12] N. Srinil and G. Rega, "The effects of kinematic condensation on internally resonant forced vibrations of shallow horizontal cables," International Journal of Non-linear Mechanics, vol. 42, no. 1, pp. 180-195, 2007.

[13] G. Rega and N. Srinil, "Nonlinear hybrid-mode resonant forced oscillations of sagged inclined cables at avoidances," Journal of Computational and Nonlinear Dynamics, vol. 2, no. 4, pp. 324-336, 2007.

[14] A. Luongo and D. Zulli, "Dynamic instability of inclined cables under combined wind flow and support motion," Nonlinear Dynamics, vol. 67, no. 1, pp. 71-87, 2012.

[15] H. Chen, D. Zuo, Z. Zhang, and Q. Xu, "Bifurcations and chaotic dynamics in suspended cables under simultaneous parametric and external excitations," Nonlinear Dynamics, vol. 62, no. 3, pp. 623-646, 2010.

[16] N. H. Krarup, Z. Zhang, and P. H. Kirkegaard, "Active modal control of rain-wind induced vibration of stay cables," Procedia Engineering, vol. 199, pp. 3158-3163, 2017.
[17] S. McTavish, A. Raeesi, A. D’Auteuil, K. Yamauchi, and H. Sato, "An investigation of the mechanisms causing largeamplitude wind-induced vibrations in stay cables using unsteady surface pressure measurements," Journal of Wind Engineering and Industrial Aerodynamics, vol. 183, pp. 19-34, 2018.

[18] H. D. Vo, H. Katsuchi, H. Yamada, and M. Nishio, "A wind tunnel study on control methods for cable dry-galloping," Frontiers of Structural and Civil Engineering, vol. 10, no. 1, pp. 72-80, 2016.

[19] K. Huang, Q. Feng, and B. Qu, "Bending aeroelastic instability of the structure of suspended cable-stayed beam," Nonlinear Dynamics, vol. 87, no. 4, pp. 2765-2778, 2017.

[20] D. Zulli and A. Luongo, "Bifurcation and stability of a twotower system under wind-induced parametric, external and self-excitation," Journal of Sound and Vibration, vol. 331, no. 2, pp. 365-383, 2012.

[21] A. Luongo and D. Zulli, "Parametric, external and self-excitation of a tower under turbulent wind flow," Journal of Sound and Vibration, vol. 330, no. 13, pp. 3057-3069, 2011.

[22] A. Luongo, G. Rega, and F. Vestroni, "Planar non-linear free vibrations of an elastic cable," International Journal of NonLinear Mechanics, vol. 19, no. 1, pp. 39-52, 1984.

[23] F. Benedettini and G. Rega, "Non-linear dynamics of an elastic cable under planar excitation," International Journal of Nonlinear Mechanics, vol. 22, no. 6, pp. 497-509, 1987.

[24] G. Rega and F. Benedettini, "Planar non-linear oscillations of elastic cables under super-harmonic resonance conditions," International Journal of Non-linear Mechanics, vol. 132, no. 3, pp. 353-366, 1989.

[25] H. M. Irvine and T. K. Caughey, "The linear theory of free vibration of suspended cable," Proceeding of Royal Society of London A, vol. 341, pp. 299-315, 1974.

[26] X. Liu, M. Zou, C. Wu, B. Yan, and M. Cai, "Galloping stability and aerodynamic characteristic of iced transmission line based on 3-DOF," Shock and Vibration, vol. 2020, no. 1, pp. 1-15, 2020.

[27] Y. Zhao, C. Sun, Z. Wang, and L. Wang, "Analytical solutions for resonant response of suspended cables subjected to external excitation," Nonlinear Dynamics, vol. 78, no. 2, pp. 1017-1032, 2014.

[28] Y. Zhao, J. Peng, Y. Zhao, and L. Chen, "Effects of temperature variations on nonlinear planar free and forced oscillations at primary resonances of suspended cables," Nonlinear Dynamics, vol. 89, no. 4, pp. 2815-2827, 2017.

[29] Q. Zhang, N. Popplewell, and A. H. Shah, "Galloping of bundle conductor," Journal of Sound and Vibration, vol. 234, no. 1, pp. 115-134, 2000.

[30] X. Liu, G. Min, C. Sun, and M. Cai, "Investigation on stability and galloping characteristics of iced quad bundle conductor," Journal of Applied Fluid Mechanics, vol. 14, no. 1, pp. 117-129, 2021.

[31] Y. Zhao, C. Huang, and L. Chen, "Nonlinear planar secondary resonance analyses of suspended cables with thermal effects," Journal of Thermal Stresses, vol. 42, no. 12, pp. 1515-1534, 2019. 